ARTICLE

\title{
Renewable fatty acid ester production in Clostridium
}

\author{
Jun Feng, 2,10, Jie Zhang1,2,10, Yuechao Ma1,2,10, Yiming Feng ${ }^{3}$, Shangjun Wang 1,2, Na Guo1,2, Haijiao Wang1,2, \\ Pixiang Wang ${ }^{1,2}$, Pablo Jiménez-Bonilla,2,4, Yanyan Gu1,2, Junping Zhou, ${ }^{1,2}$, Zhong-Tian Zhang1,2,

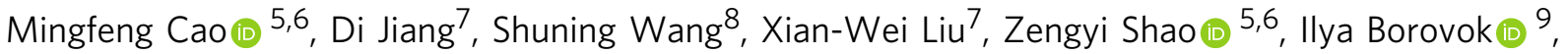 \\ Haibo Huang ${ }^{3 凶} \&$ Yi Wang (i) ${ }^{1,2 凶}$
}

Bioproduction of renewable chemicals is considered as an urgent solution for fossil energy crisis. However, despite tremendous efforts, it is still challenging to generate microbial strains that can produce target biochemical to high levels. Here, we report an example of biosynthesis of high-value and easy-recoverable derivatives built upon natural microbial pathways, leading to improvement in bioproduction efficiency. By leveraging pathways in solventogenic clostridia for co-producing acyl-CoAs, acids and alcohols as precursors, through rational screening for host strains and enzymes, systematic metabolic engineering-including elimination of putative prophages, we develop strains that can produce $20.3 \mathrm{~g} / \mathrm{L}$ butyl acetate and $1.6 \mathrm{~g} / \mathrm{L}$ butyl butyrate. Techno-economic analysis results suggest the economic competitiveness of our developed bioprocess. Our principles of selecting the most appropriate host for specific bioproduction and engineering microbial chassis to produce high-value and easy-separable end products may be applicable to other bioprocesses.

\footnotetext{
${ }^{1}$ Department of Biosystems Engineering, Auburn University, Auburn, AL, USA. ${ }^{2}$ Center for Bioenergy and Bioproducts, Auburn University, Auburn, AL, USA ${ }^{3}$ Department of Food Science and Technology, Virginia Tech, Blacksburg, VA, USA. ${ }^{4}$ School of Chemistry, National University (UNA), Heredia, Costa Rica.

${ }^{5}$ Department of Chemical and Biological Engineering, lowa State University, Ames, IA, USA. ${ }^{6}$ NSF Engineering Research Center for Biorenewable Chemicals, lowa State University, Ames, IA, USA. ${ }^{7}$ Department of Applied Chemistry, University of Science and Technology of China, Hefei, China. ${ }^{8}$ State Key Laboratory of Microbial Technology, Microbial Technology Institute, Shandong University, Qingdao, China. ${ }^{9}$ The Shmunis School of Biomedicine and Cancer Research, Faculty of Life Sciences, Tel Aviv University, Ramat Aviv, Tel Aviv, Israel. ${ }^{10}$ These authors contributed equally: Jun Feng, Jie Zhang, Yuechao Ma.

凶email: huang151@vt.edu; yiwang3@auburn.edu
} 
A lthough tremendous efforts have been invested for biofuel and biochemical research, it is still challenging to generate robust microbial strains that can produce target products at desirable levels ${ }^{1}$. We hypothesize that, the production of high-value bioproducts which can be easily recovered from fermentation might be a solution to tackle the bottleneck in bioproduction. Fatty acid esters, or mono-alkyl esters, can be used as valuable fuels such as diesel components or specialty chemicals for food flavoring, cosmetic and pharmaceutical industries ${ }^{2,3}$. It is projected that the US market demand for fatty acid esters could reach $\$ 4.99$ billion by $2025^{4}$. In addition, esters, with fatty acid and alcohol moieties, are generally hydrophobic and can be easily separated from fermentation, leading to high bioproduction efficiency.

Conventionally, esters are produced through Fischer esterification which involves high temperature and inorganic catalysts $^{5,6}$. The reaction consumes a large amount of energy and generates tremendous wastes, and thus is not environmentally friendly ${ }^{5}$. On the other hand, ester production through biological routes is becoming more and more attractive because it is renewable and environmentally benign ${ }^{3}$. There are two primary biological pathways for ester production: one is through esterification of fatty acid and alcohol catalyzed by lipases 3,7 , and the other is based on condensation of acyl-CoA and alcohol catalyzed by alcohol acyl transferases (AATs) ${ }^{5}$. Previously, lipases from bacteria or fungi have been employed for catalyzing esterification for ester production ${ }^{8}$. For instance, lipase from Candida $s p$. has been recruited to drive production of butyl butyrate (BB) with Clostridium tyrobutyricum. C. tyrobutyricum, a natural hyperbutyrate producer, could generate $34.7 \mathrm{~g} / \mathrm{L}$ BB with supplementation of lipase and butanol ${ }^{9}$. However, in such a process, the supplemented enzyme accounts for a big cost and meanwhile the operation needs to be carefully managed to achieve the optimum performance of esterification ${ }^{9}$. Therefore, a whole microbial cell factory capable of ester production in one pot is highly desired. Some microorganisms can naturally produce esters ${ }^{3}$. For instance, Kluyveromyces marxianus DSM 5422 has been reported to produce $8.92 \mathrm{~g} / \mathrm{L}$ of ethyl acetate (EA) in the fermentation ${ }^{10}$. Saccharomyces cerevisiae could also produce various esters with its native AATs, but generally at very low levels $(<1 \mathrm{~g} / \mathrm{L})^{11}$. Metabolic engineering of microorganisms for ester production by introducing the heterologous ester-producing pathways has been attempted by various researchers. Horton et al. introduced the AAT genes of $S$. cerevisiae into $C$. acetobutylicum and Escherichia coli and obtained $0.6 \mathrm{mM}$ and $1.8 \mathrm{mM}$ isoamyl acetate, respectively ${ }^{12}$. The same group of researchers also achieved the production of small amount of EA, butyl acetate (BA) and BB when they introduced AAT genes from S. cerevisiae and strawberry into E. coli ${ }^{13}$. Bohnenkamp et al. engineered $E$. coli to produce up to $3.8 \mathrm{~g} / \mathrm{L}$ EA by overexpressing the eat 1 gene from Wickerhamomyces anomalus ${ }^{14}$. Rodriguez et al. metabolically engineered $E$. coli to produce esters by introducing heterologous $\mathrm{AATs}^{5}$. Although the production of some acetate esters can reach decent levels (such as 17.2 $\mathrm{g} / \mathrm{L}$ isobutyl acetate), the production of most of the esters was rather low, probably due to the unavailability of intrinsic substrates/precursors and limited tolerance of E. coli to organic endproducts.

In this work, we report highly efficient fatty acid ester production to high levels using engineered clostridia. We select solventogenic clostridia to take advantage of their natural pathways for co-producing acyl-CoAs (acetyl-CoA and butyryl-CoA), fatty acids (acetate and butyrate), and alcohols (ethanol and butanol), either as intermediates or endproducts; in addition, solventogenic clostridia tend to possess good tolerance to toxic endproducts thanks to their intrinsic capacity for high-level solvent production. We hypothesize that clostridia can be excellent microbial platforms to be engineered for efficient ester production by introducing heterologous AATs and/or lipase genes. Indeed, through rational screening for host strains (from four well-known clostridial species) and enzymes (AATs and lipase), systematic metabolic engineering-including rational organization of estersynthesizing enzymes inside of the cell, and elimination of the prophage-like regions, we ultimately obtain two strains which can produce $20.3 \mathrm{~g} / \mathrm{L} \mathrm{BA}$ and $1.6 \mathrm{~g} / \mathrm{L} \mathrm{BB}$ in extractive batch fermentations.

\section{Results}

Screening of host strains and genes for ester production. We considered clostridia as ideal platforms for ester production thanks to their intrinsic intermediates (fatty acids, acyl-CoAs, and alcohols) serving as precursors for ester biosynthesis (Fig. 1). We hypothesized that different flux levels of these precursors within various clostridial strains would make a big difference for the specific type(s) of ester production. Therefore, we selected five strains (from four representative species) including $C$. tyrobutyricum $\Delta$ cat $1:: a d h E 1^{15}$, C. tyrobutyricum $\Delta$ cat $1:: a d h E 2^{15}, C$. pasteurianum SD-1 ${ }^{16}$, C. saccharoperbutylacetonicum N1-4-C ${ }^{17}$ and C. beijerinckii NCIMB $8052^{18}$ to evaluate their capabilities for ester production through metabolic engineering. We included both $C$. tyrobutyricum $\Delta c a t 1:: a d h E 1$ and $\Delta c a t 1:: a d h E 2$ here because they produce different levels (and thus ratios) of butanol and ethanol ${ }^{15,19}$. Esters can be synthesized either through esterification of acid and alcohol catalyzed by lipase, or through condensation of acyl-CoA and alcohol catalyzed by AATs (Fig. 1). Previously, lipase B (CALB) from Candida antarctica has been employed for efficient ester production through esterification ${ }^{9,20}$. In addition, four AATs including $\mathrm{VAAT}^{23}, \mathrm{SAAT}^{21,23}, \mathrm{ATF}^{2,11}$, $\mathrm{EHT}^{22}$ have been recruited for ester production in various hosts ${ }^{5,23-25}$. Therefore, here, we evaluated all these genes in our clostridial hosts for ester production.

Six plasmids (pMTL- $\mathrm{P}_{\text {cat }}$-vaat, pMTL- $\mathrm{P}_{\text {cat }}$-saat, pMTL- $\mathrm{P}_{\text {cat }}{ }^{-}$ atf1, pMTL- $\mathrm{P}_{\text {cat }}$-eht1, pMTL- $\mathrm{P}_{\text {cat }}$-lipaseB as well as pMTL82151 as the control) were individually transformed into $C$. saccharoperbutylacetonicum N1-4-C, C. pasteurianum SD-1, C. tyrobutyricum $\Delta$ cat $1:: a d h E 1$ and $\Delta$ cat $1:: a d h E 2$, respectively. While

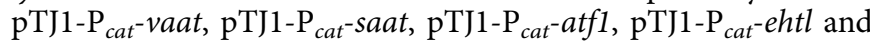
pTJ1- $\mathrm{P}_{\text {cat }}$-lipaseB as well as pTJ1 were transformed into $C$. beijerinckii 8052. Fermentations were performed (Fig. 2a), and results were shown in Fig. 2b. Four types of esters were detected: EA, BA, ethyl butyrate (EB) and BB. Interestingly, control strains with the empty plasmid (pMTL82151 or pTJ1) also produced noticeable EA, BA and BB. This could be because: (1) the endogenous lipase in clostridia can catalyze ester production ${ }^{17}$; (2) catP on pMTL82151 encoding a chloramphenicol acetyltransferase (belonging to the same class of enzymes as AATs) has AAT activities 5,26 .

Based on the results, it could be concluded that ATF1 is more favorable for BA production. All strains with atf1 produced higher levels of BA compared to the same strain but with the overexpression of other genes (Fig. 2b). While VAAT, SAAT and EHT1 seemed to have better activities for $\mathrm{BB}$ production. Among all the strains, C. saccharoperbutylacetonicum FJ-004 produced the titer of $5.5 \mathrm{~g} / \mathrm{L}$ BA. Previously, C. acetobutylicum CaSAAT (with the overexpression of saat from Fragaria xananassa) was reported to produce $8.37 \mathrm{mg} / \mathrm{L}$ $\mathrm{BA}^{25}$. While this manuscript was prepared, a newly engineered C. diolis strain was reported to produce $1.37 \mathrm{~g} / \mathrm{L} \mathrm{BA}^{27}$. Here, the $\mathrm{BB}$ production of $0.3 \mathrm{~g} / \mathrm{L}$ was observed in C. pasteurianum $\mathrm{J}-5$ with the overexpression of eht 1 , which is higher than the recently reported $50.07 \mathrm{mg} / \mathrm{L}$ in an engineered C. acetobutylicum ${ }^{25}$. Six of our engineered strains could also produce small amount of EB with the titer up to $0.02 \mathrm{~g} / \mathrm{L}$ been observed in C. pasteurianum J-5. With the 


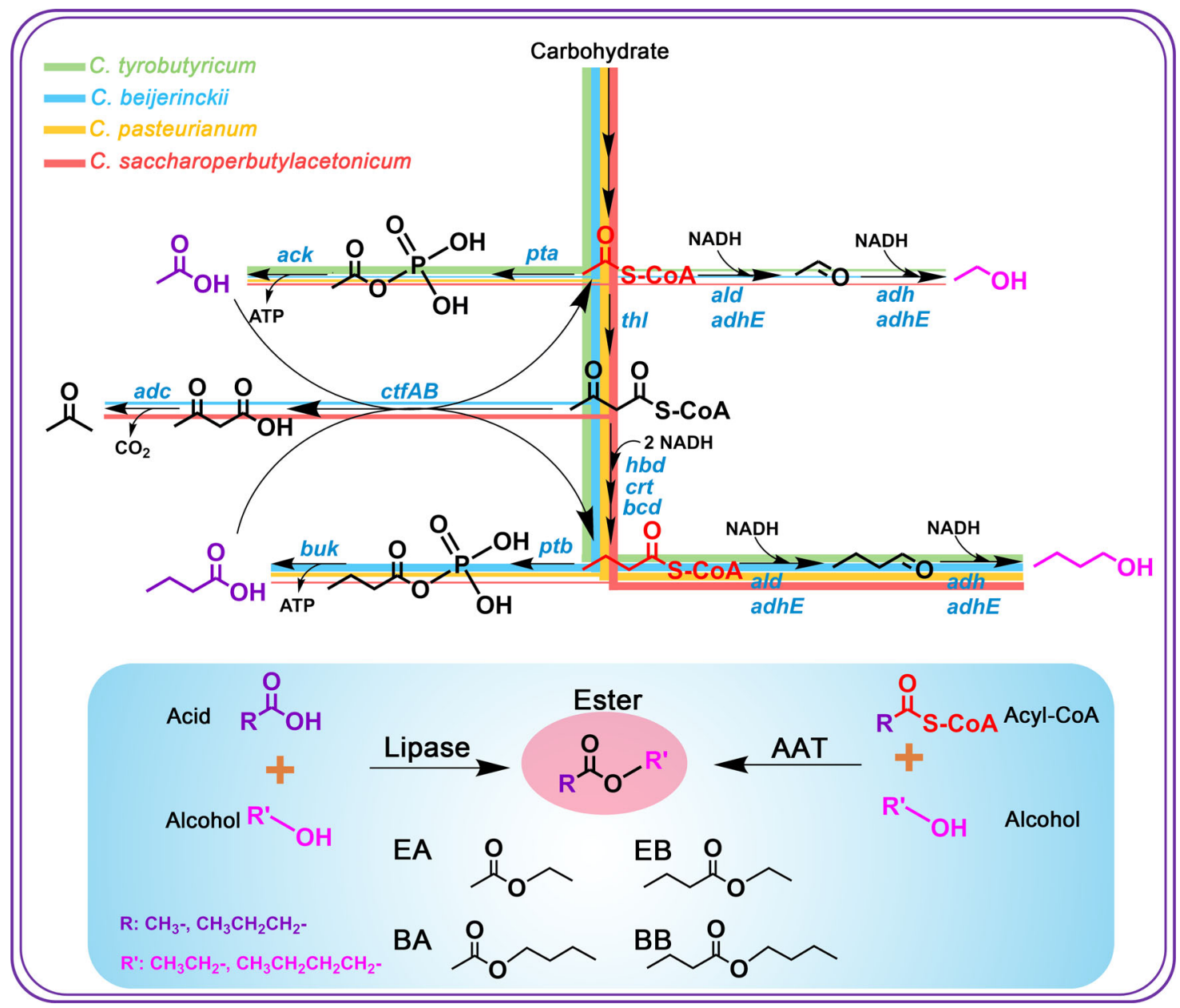

Fig. 1 Engineering of solventogenic clostridia for fatty acid ester production. Top: Five strains out of four representative clostridial species were selected and evaluated as the host to be engineered for ester production in this study. We hypothesized that the different metabolic fluxes within different strains would make a big difference for the desirable ester production. The metabolic pathways of the four different species were represented in four different colors. Bottom: Fatty acid esters could be synthesized through two primary biological pathways: one is through the esterification of fatty acid and alcohol catalyzed by lipases, and the other is through the condensation of acyl-CoA and alcohol catalyzed by alcohol acyl transferases (AATs). Key genes in the pathway: pta phosphotransacetylase, ack acetate kinase, thl thiolase, hbd beta-hydroxybutyryl-CoA dehydrogenase, crt crotonase, bcd butyryl-CoA dehydrogenase, adh alcohol dehydrogenase, adhE aldehyde-alcohol dehydrogenase, adc acetoacetate decarboxylase, ctfAB CoA transferase, ptb phosphotransbutyrylase, buk butyrate kinase, ald aldehyde dehydrogenase. EA: ethyl acetate, EB: ethyl butyrate, BA: butyl acetate, BB: butyl butyrate.

lipaseB overexpression, C. tyrobutyricum JZ-6 could generate $0.3 \mathrm{~g} / \mathrm{L}$ EA. This was significantly higher than other strains tested in this work $($ mostly $<0.01 \mathrm{~g} / \mathrm{L})$. As we reported previously, the mother strain C. tyrobutyricum $\Delta$ cat $1:: a d h E 1$ could produce $20.8 \mathrm{~g} / \mathrm{L}$ acetate and $5.3 \mathrm{~g} / \mathrm{L}$ ethanol (precursors for EA synthesis) during a batch fermentation, which might have enabled high-level EA production in C. tyrobutyricum $\mathrm{JZ}-6^{15}$.

The production levels of $\mathrm{BA}$ and $\mathrm{BB}$ achieved above are both significantly higher than the previously reported in microbial hosts. In comparison, the BA level is much higher than BB level, and thus has greater potentials toward economic viability. Therefore, in the following steps, we primarily focused on systematic metabolic engineering of the strain for further enhanced BA production.

Enhancement of NADH availability. Based on the pathway, for either butanol (the precursor for BA production) or BA production, a large amount of reducing power (NADH) is required. Therefore, we set out to increase the availability of $\mathrm{NADH}$ to improve BA production. It has been reported that in the solventogenic $C$. acetobutylicum cells, the $\mathrm{NADPH} / \mathrm{NADP}^{+}$ratio is at least 70 times higher than the NADH/NAD ${ }^{+}$ratio $^{28,29}$.
It therefore indicates in a sense that there is surplus NADPH available that can be leveraged for enhanced production of reduced endproducts. Indeed, by overexpressing a gene encoding a mutated aldehyde/alcohol dehydrogenase that possesses increased affinity for NADPH, Jang et al. significantly improved the butanol production in an engineered $C$. acetobutylicum strain $^{30}$. In another study, by replacing the NADH-dependent hbd (encoding beta-hydroxybutyryl-CoA dehydrogenase) in C. acetobutylicum with the strictly NADPH-dependent $h b d 1$ from C. kluyveri, butanol/ethanol ratio and butanol flux were improved by 6- and 1.6-fold, respectively; meanwhile, the butanol yield reached $0.34 \mathrm{~g} / \mathrm{g}$ in a simple mineral media without an organic nitrogen source ${ }^{28}$.

On the other hand, the recently discovered flavin-based electron-bifurcating NADH-dependent reduced ferredoxin: $\mathrm{NADP}^{+}$oxidoreductase $\mathrm{NfnAB}$ catalyzes the transhydrogenation reaction among $\mathrm{NAD}^{+}, \mathrm{NADP}^{+}$, and ferredoxin ${ }^{31}$. Electronbifurcating $\mathrm{Nfn}$ widely exists in anaerobes such as solventogenic clostridia, and catalyzes the endergonic reduction of $\mathrm{NADP}^{+}$with $\mathrm{NADH}$ coupled to the exergonic reduction of $\mathrm{NADP}^{+}$with reduced ferredoxin under appropriate physiological conditions. 


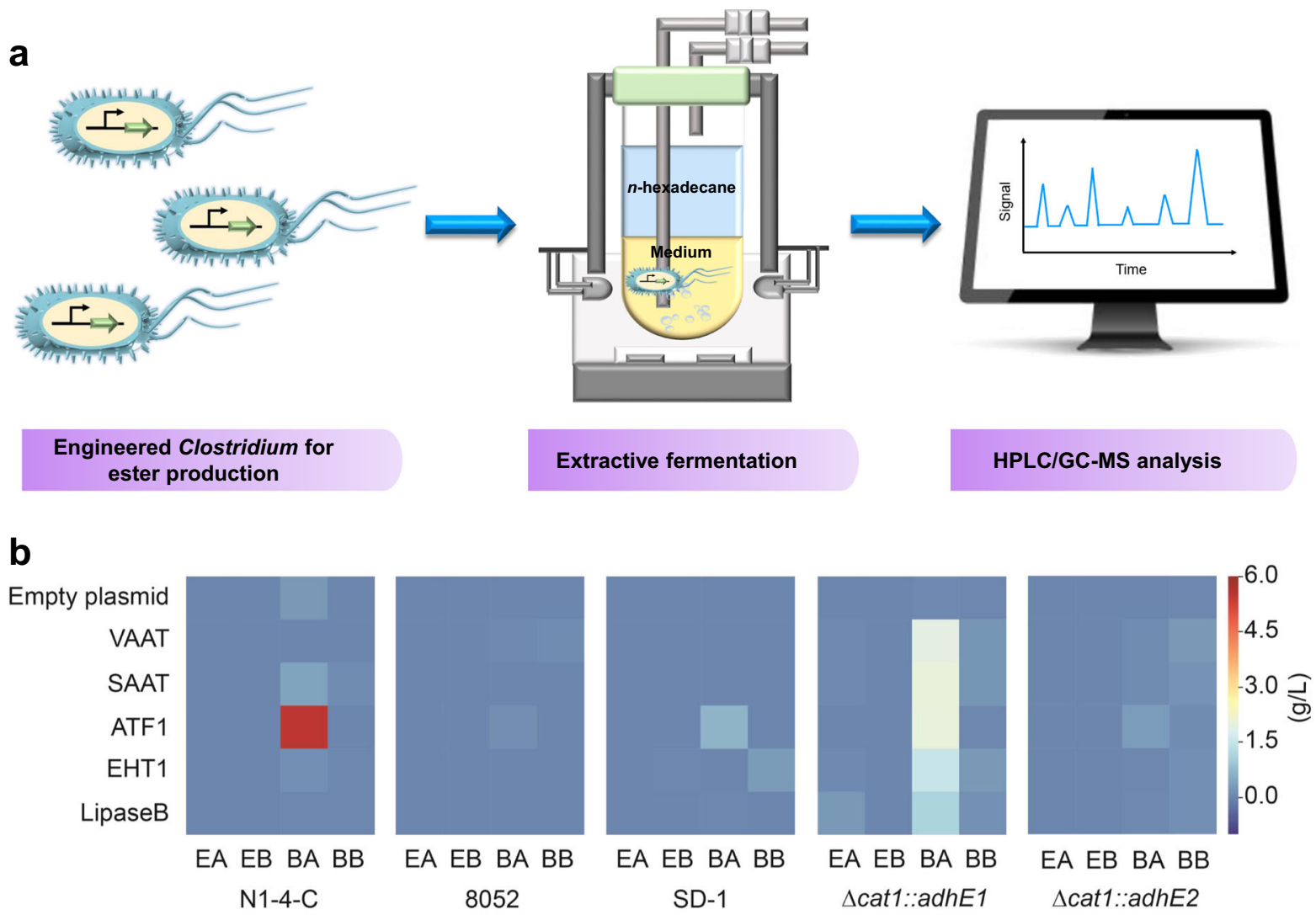

Fig. 2 Screening of strains and enzymes for ester synthesis. a Schematic representation of procedures for extractive ester fermentation. b Heatmap results showed the ester production in various Clostridium strains with the overexpression of different enzymes. Empty plasmid: expression of pMTL82151 (for C. saccharoperbutylacetonicum N1-4-C, C. pasteurianum SD-1, C. tyrobutyricum cat1::adhE1 and cat1::adhE2) or pTJ1 (for C. beijerinckii 8052) as the control; EA: ethyl acetate, EB: ethyl butyrate, BA: butyl acetate, BB: butyl butyrate. Source data underlying Fig. $2 \mathrm{~b}$ are provided as a Source Data file.

The ferredoxin-dependent transhydrogenase plays an important role in maintaining the redox balance in vivo, and participates in various metabolisms including acetogenesis, butyrate formation, and $\mathrm{H}_{2}$ production ${ }^{32}$. In this study, we hypothesized that the gene cluster of Cspa_c47560/Cspa_c47550 encodes an enzymatic complex possessing a function similar to $\mathrm{NfnAB}$ for $\mathrm{NADH}$ dehydrogenation and ferredoxin oxidation (see Supplementary Note 1, Supplementary Method 1), and, by deleting it (in full or partially), butanol (in butanol-producing mother strain) and BA (in BA-producing mutant) production would be boosted because of the potentially increased NADH availability (by decreasing the conversion of NADH to NADPH). Thus, we deleted Cspa_c47560 in N1-4-C and generated FJ-100. Further, FJ-101 was constructed based on FJ-100 for BA production. Results demonstrated that, although butanol production in FJ-100 was only slightly improved (16.5 g/L vs. 15.8 g/L in N1-4-C; Supplementary Fig. 1), BA production in FJ-101 was remarkably enhanced compared to FJ-004 (7.8 g/L vs. $5.5 \mathrm{~g} / \mathrm{L})$. Based on our experiences, because $C$. saccharoperbutylacetonicum N1-4 (HMT) (or N1-4-C) mother strain can naturally produce very high level of butanol, it was generally very difficult to further improve butanol production in C. saccharoperbutylacetonicum through simple metabolic engineering strategies ${ }^{33}$. This is likely the case in FJ-100 (comparing to N1-4-C). However, the increased NADH availability with Cspa_c47560 deletion in FJ-101 (see Supplementary Note 1 and Supplementary Fig. 2) would enable an enhanced instant flux/ availability of butanol which would serve as the precursor for BA production and thus enhance BA production in FJ-101. In this sense, the total butanol generated during the process (including the fraction serving as the precursor for BA production and the other fraction as the endproduct) in FJ-101 would be actually much higher than in FJ-004.

Tuning of acetyl-CoA availability/flux. Besides butanol, acetyl$\mathrm{CoA}$ is the other primary precursor for BA production. We set out with two strategies to attune the acetyl-CoA availability/flux in attempt to further enhance BA production: (1) to decrease the flux of acetyl-CoA to acetoacetyl-CoA by deleting the thiolase gene, and (2) to pull the metabolic flux to acetone to enhance the acetate reassimilation (and thus the acetyl-CoA regeneration) by integrating the pathway of converting acetone into isopropanol (Fig. 3a). While we initially failed with (1) despite multiple trials (see Supplementary Note 2), we easily achieved (2) as we did previously when we engineered $C$. saccharoperbutylacetonicum for high level isopropanol-butanol-ethanol (IBE) production as also described below ${ }^{34}$.

The sadh gene in C. beijerinckii B-593 encoding a secondary alcohol dehydrogenase can convert acetone into isopropanol ${ }^{35}$. The hydG gene in the same operon as sadh encodes a putative electron transfer protein. It has been demonstrated to play important roles for the conversion of acetone into isopropanol ${ }^{34,36}$. In this study, either $s a d h$ alone or the sadhhydG gene cluster was integrated into the chromosome of FJ-100, generating FJ-200 and FJ-300, respectively. Further, by introducing pMTL-cat-atf1, FJ-201 and FJ-301 were obtained. Compared to FJ-101, about $50 \%$ of the acetone could be converted into isopropanol in FJ-201, while $~ 95 \%$ of the acetone in FJ-301 could be converted into isopropanol. The total titers of acetone plus isopropanol in FJ-301 and FJ-201 were $6.6 \mathrm{~g} / \mathrm{L}$ and $5.1 \mathrm{~g} / \mathrm{L}$ respectively, both of which were higher than $4.8 \mathrm{~g} / \mathrm{L}$ acetone in 
a

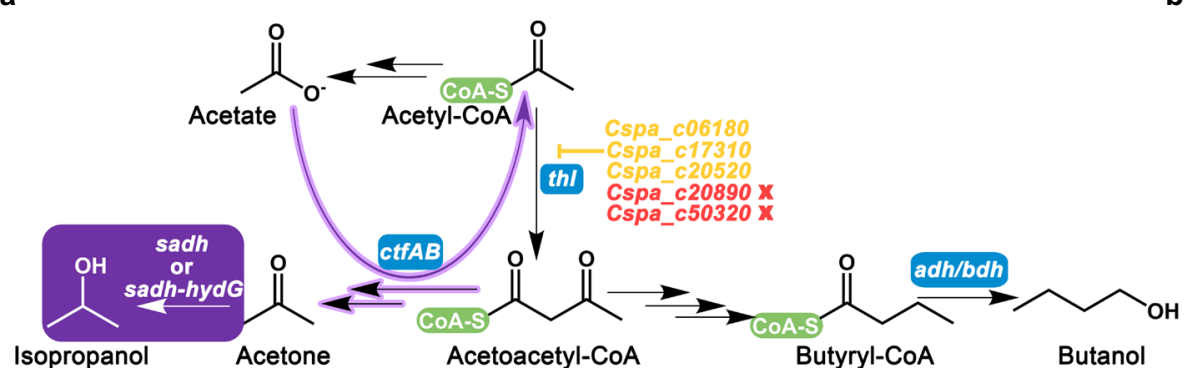

b

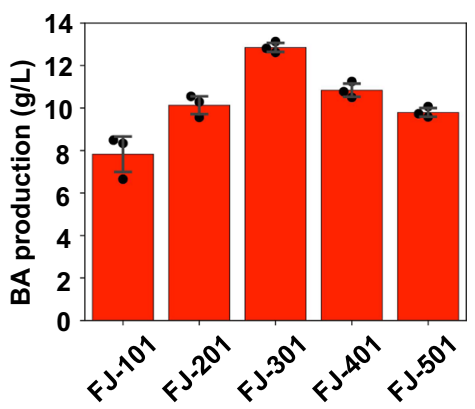

C

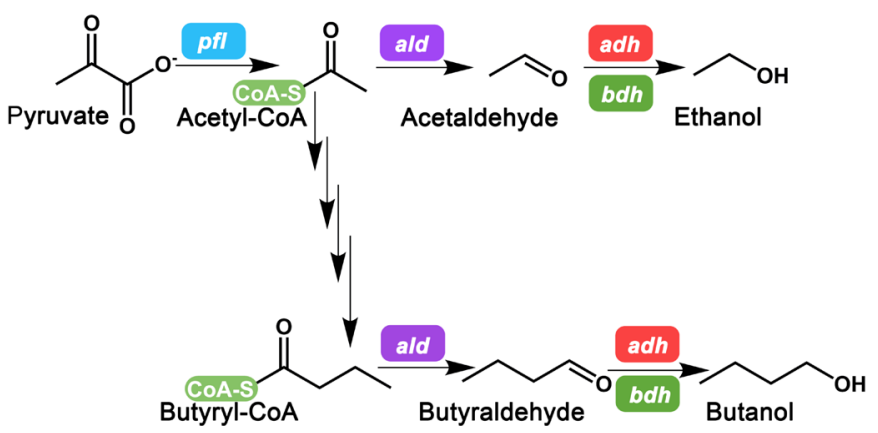

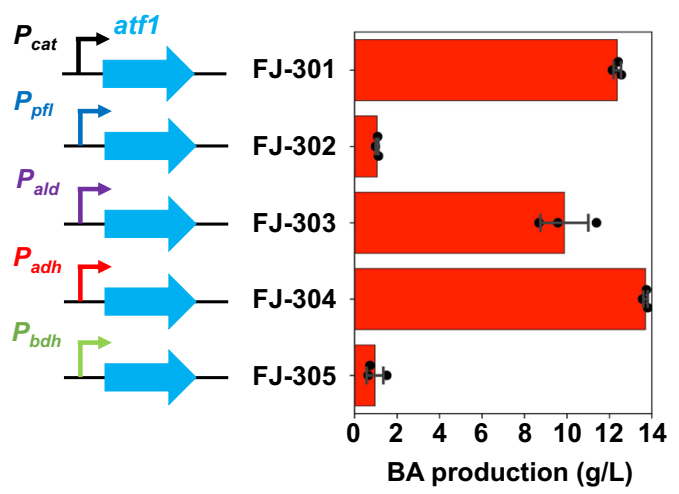

Fig. 3 Enhancing butyl acetate production through increasing the availability of acetyl-CoA and dynamically expressing the atf1 gene. a Introduction of isopropanol synthesis pathway (shaded in purple) and deletion of thiolase genes. The pathways in purple arrows represent the regeneration of acetyl-CoA. There are five annotated genes encoding thiolase in C. saccharoperbutylacetonicum, only two (in red) of which could be deleted (see Supplementary Note 2). b The fermentation results for butyl acetate (BA) production with various mutant strains corresponding to the genetic manipulations in (a). Data are presented as mean values $\pm \mathrm{SD}(n=3)$. c Four promoters associated with the biosynthesis of acetyl-CoA or alcohols in the pathway were selected to drive the expression of atf1. d The fermentation results for BA production with various mutant strains in which different promoters were used to drive the expression of atf1 as illustrated in (c). Data are presented as mean values \pm SD $(n=3)$. Key genes in the pathway: adh alcohol dehydrogenase, bdh butanol dehydrogenase, $\operatorname{ct} f A B$ CoA transferase, sadh secondary alcohol dehydrogenase, hydG putative electron transfer protein, pfl pyruvate formate lyase, ald aldehyde dehydrogenase. Source data underlying Fig. $3 b$ and $d$ are provided as a Source Data file.

FJ-101. More significantly, BA production in FJ-201 and FJ-301 has been remarkably increased compared to FJ-101, and reached $10.1 \mathrm{~g} / \mathrm{L}$ and $12.9 \mathrm{~g} / \mathrm{L}$, respectively (Fig. 3b). This result is somewhat surprising. One possible reason could be because isopropanol is less toxic than acetone to the cells; however, our cell growth tolerance experiments ruled out this possibility (Supplementary Note 2, Supplementary Fig. 3, and Supplementary Method 2). Another possible explanation is that, by pulling flux from acetone to isopropanol, the transferring of CoA from acetoacetyl-CoA to acetate driven by the CoA transferase would be boosted, resulting in increased instant availability of acetyl$\mathrm{CoA}$, and thus enhanced BA production (also see Supplementary Note 2). Anyway, it warrants a further investigation on this matter through, for example, a systematic metabolic flux analysis and/or metabolic modelling analysis.

Dynamic expression of atf1. In our engineered strain, BA is synthesized through condensation of butanol and acetyl-CoA catalyzed by ATF1. The constitutively high expression of ATF1 would not necessarily lead to high BA production. For example, BA production in FJ-008 in which atf1 was driven by the constitutive strong promoter $\mathrm{P}_{t h l}$ from $C$. tyrobutyricum was actually much lower $(3.5 \mathrm{~g} / \mathrm{L}$ vs. $5.5 \mathrm{~g} / \mathrm{L})$ than in FJ-004 in which atf1 was expressed under the promoter $\mathrm{P}_{c a t}$ from $C$. tyrobutyricum. We hypothesized that, in order to obtain more efficient BA production, the synthesis of ATF1 should be dynamically controlled and thus synchronous with the synthesis of precursors (butanol or acetyl-CoA). Therefore, for the next step, we attempted to evaluate various native promoters for atf1 expression, and identify the one(s) that can enable an appropriately dynamic expression of ATF1 and lead to enhanced BA production.

Four promoters associated with the synthesis of BA precursors were selected to drive the atf1 expression, and four strains were constructed correspondingly for BA production (Fig. 3c). Fermentation results were shown in Fig. 3d. Indeed, distinct results for BA production were observed in these strains. The BA level was only 1.0 and $1.1 \mathrm{~g} / \mathrm{L}$ in FJ-305 and FJ-302 with $\mathrm{P}_{b d h}$ and $\mathrm{P}_{p f l}$ for atfl expression, respectively. $\mathrm{P}_{\text {ald }}$ is an important promoter in $C$. saccharoperbutylacetonicum, which can sense the acidic state and switch cell metabolism from acidogenesis to solventogenesis ${ }^{37}$. FJ303 with the atf1 expression driven by $\mathrm{P}_{\text {ald }}$ produced $9.9 \mathrm{~g} / \mathrm{L} \mathrm{BA}$, which was still $20 \%$ lower than in FJ-301. Interestingly, FJ-304 in which atf1 was expressed under $\mathrm{P}_{a d h}$ produced $13.7 \mathrm{~g} / \mathrm{L} \mathrm{BA}$, which was about $10.5 \%$ higher than in FJ-301. Based on the results, we speculated that the promoter of adh (which encodes the key enzyme catalyzing butanol and ethanol production) might have resulted in the more appropriate dynamic expression of atf 1 in line with the flux of the precursors and thus led to enhanced BA production. On the other hand, ethanol production in FJ-304 was also lower than in FJ-301 (0.3 g/L vs. $0.6 \mathrm{~g} / \mathrm{L})$. The enhanced BA production in FJ-304 consumed more acetyl-CoA, and thus decreased production of ethanol, which also needed acetyl-CoA as the precursor.

Rational organization of BA-synthesis enzymes. Rational organization of enzymes associated with the synthesis of target product is an effective strategy to improve the bioproduction ${ }^{38}$. In 
a
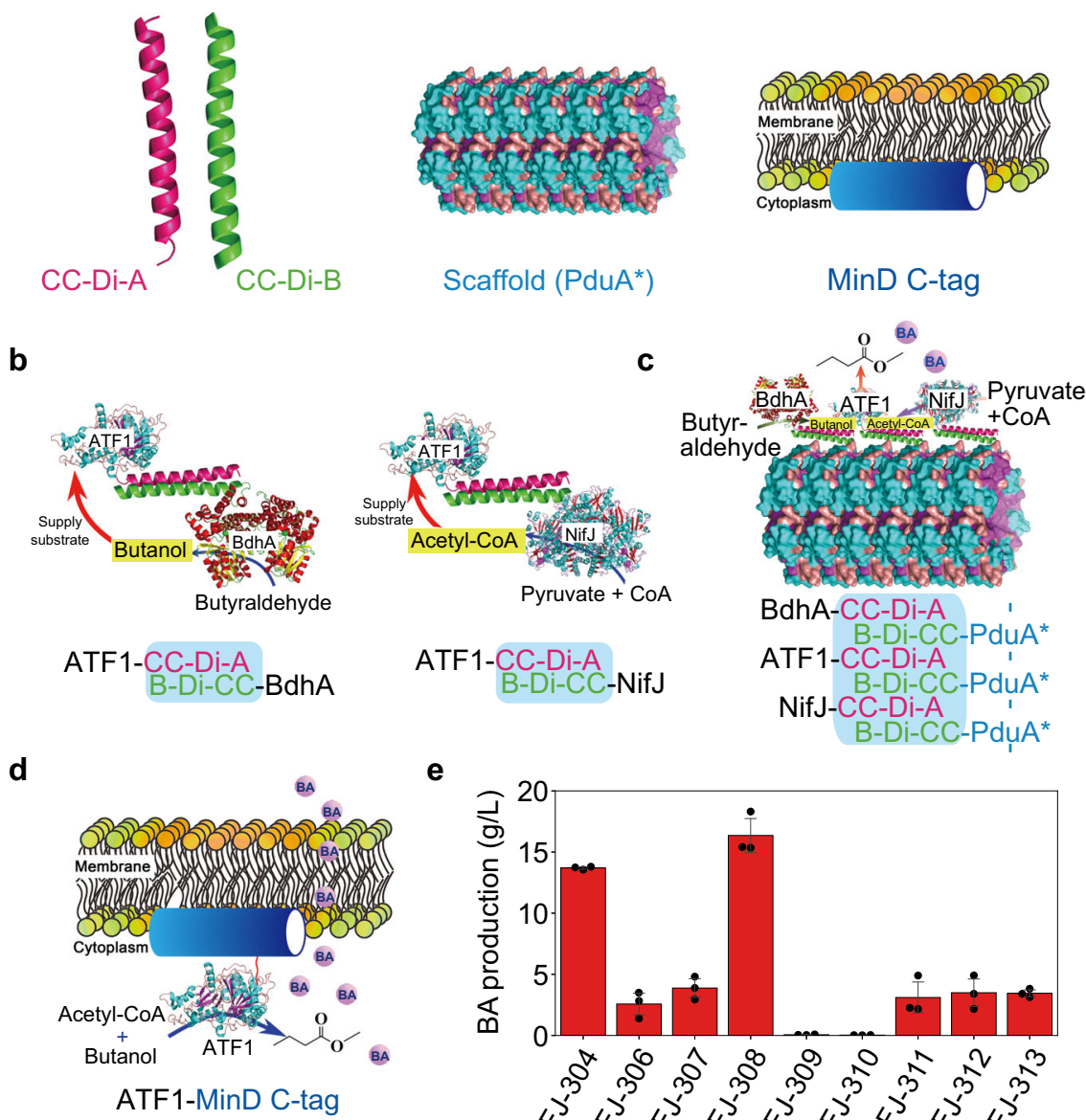

e

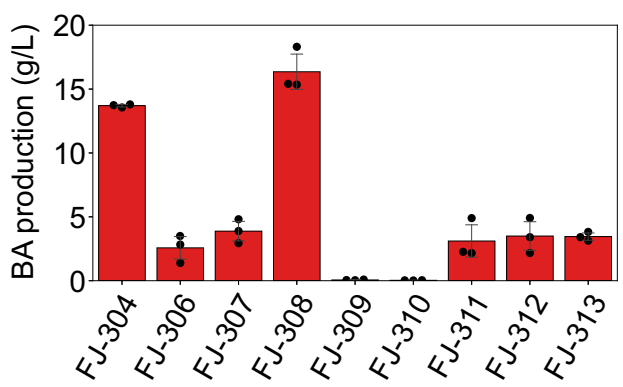

Fig. 4 Enhancing butyl acetate production through rational organization of the enzymes associated with BA synthesis. a Biological components evaluated in this study for rational organization of enzymes: CC-Di-A and CC-Di-B tags, PduA* scaffold, and MinD C-tag. $\mathbf{b}$ Schematic representation of assembling two of the three enzymes for BA synthesis with the CC-Di-A and CC-Di-B tags. c Schematic representation of organizing the three enzymes for BA synthesis onto the PduA* formed scaffold. d Schematic representation of using the MinD C-tag to draw ATF1 onto the cell membrane. e The fermentation results for butyl acetate (BA) production with various mutant strains corresponding to the genetic manipulations from (b-d). Data are presented as mean values \pm SD $(n=3)$. Source data underlying Fig. 4 e are provided as a Source Data file.

this study, we evaluated several such approaches to increase BA production (Fig. 4).

$\mathrm{PduA}^{*}$ protein, derived from Citrobacter freundii Pdu bacterial microcompartment could form filaments in bacteria like E. coli ${ }^{38}$. The CC-Di-A and CC-Di-B are designed parallel heterodimeric coiled coils and two proteins with each of these self-assembling tags could combine and shorten the catalytic distance. The enzymes (from the same metabolic pathway), tagged with one of the coiled coils (CC-Di-A or CC-Di-B) would attach onto the formed intracellular filaments (its $\mathrm{PduA}^{*}$ was tagged by the other coiled coil); thus, the organized enzymes on the filaments would improve the catalytic efficiency of the target metabolic pathway. MinD is a membrane-associated protein and the localization of $\mathrm{MinD}$ is mediated by an 8-12 residue C-terminal membrane-targeting sequence. The proteins with MinD C-terminal sequence were able to be drawn to the cell membrane ${ }^{38,39}$. Thus, the application of MinD C-tag can facilitate the secretion of target product and enhance its production by mitigating the intracellular toxicity as well as promoting the catalyzing process (Fig. 4a).

To evaluate whether the organization of enzymes could improve BA production in our strain, three strategies were recruited: (1) assembling the enzymes associated with BA synthesis: NifJ (related to acetyl-CoA synthesis), BdhA (related to butanol synthesis) and ATF1 with the CC-Di-A and/or CC-Di-B tags (FJ-
306, FJ-309 and FJ-310); (2) organizing the three enzymes onto PduA* formed scaffold (FJ-311, FJ-312 and FJ-313); or (3) introducing MinD C-tag to draw ATF1 onto the cell membrane (FJ-307, FJ-308).

Firstly, we assembled enzymes for BA synthesis by adding the CC-Di-A tag to ATF1 and the CC-Di-B tag to NifJ and BdhA (Fig. 4b). Fermentation results showed that the addition of CCDi-A tag to the C-terminus of ATF1 in FJ-306 had significantly negative effects on BA synthesis with only $2.6 \mathrm{~g} / \mathrm{L}$ BA was produced (Fig. 4e). The assembly of ATF1 together with NifJ, or NifJ and BdhA, had even severer negative effects on BA synthesis and BA production was only 0.06 and $0.03 \mathrm{~g} / \mathrm{L}$ in FJ-309 and FJ310, respectively. Further, we organized ATF1, NifJ and BdhA onto the PduA* scaffold. PduA* was tagged with CC-Di-B, while the other three enzymes were tagged with CC-Di-A (Fig. 4c). The generated FJ-311 (harboring the scaffold and ATF1-CC-Di-A) produced 3.1 g/L BA, while FJ-312 (harboring the scaffold, ATF1CC-Di-A and NifJ-CC-Di-A) and FJ-313 (harboring the scaffold, ATF1-CC-Di-A, NifJ-CC-Di-A and BdhA-CC-Di-A) produced slightly higher amount of BA both at $3.5 \mathrm{~g} / \mathrm{L}$. The scaffold seemed to have some positive effects on BA synthesis but didn't work as efficient as it was reported in other studies ${ }^{38}$. We speculate that the coiled coils tags (CC-Di-A or CC-Di-B) might severely impair the catalytic activity of ATF1. Furthermore, the assembly of ATF1 
together with Niff, or NifJ and BdhA, could further inhibit the activity of ATF1 and significantly impair the cell growth (FJ-309 and FJ-310 demonstrated lowest cell growth among all these strains; see Supplementary Note 3 and Supplementary Fig. 4), thus resulting in significant decrease in BA production in the corresponding strains.

Moreover, we evaluated the effect of the introduction of MinD C-tag (to draw ATF1 onto the cell membrane) on BA production (Fig. 4d). Fermentation results showed that the corresponding FJ308 strain could produce $16.4 \mathrm{~g} / \mathrm{L} \mathrm{BA}$, which was $20 \%$ higher than FJ-304 (Fig. 4e). The FJ-307 strain (with CC-Di-A-ATF1-MinD) could also produce higher BA of $3.9 \mathrm{~g} / \mathrm{L}$ compared to FJ-306 $(2.6 \mathrm{~g} / \mathrm{L})$. All these results indicated that the addition of the cell membrane-associated motif to draw ATF1 onto the cell membrane could facilitate the BA excretion and mitigate the intracellular toxicity and therefore enhance $\mathrm{BA}$ production (Supplementary Note 3, Supplementary Fig. 5, and Supplementary Method 3). However, the assembly of BA synthetic enzymes would significantly decrease BA production.

Elimination of prophages. During our fermentations, we noticed that the ester production of the strains was not stable and could be varied from batch to batch. It has been reported decades ago that the former strain N1-4 (ATCC 13564) was lysogenized with a temperate phage HM T, generating a new strain N1-4 (HMT), which could release phage particles from the chromosome even without induction ${ }^{40}$. In addition, the N1-4 (HMT) strain can produce a phage-like bacteriocin Clostocin O upon the induction with mitomycin $\mathrm{C}^{41}$. We hypothesized that the instability of fermentations with $C$. saccharoperbutylacetonicum might be related to the prophages, and the deletion of prophages would enable more stable and enhanced production of desired endproducts. Our in silico analysis of the N1-4 (HMT) genome revealed five prophage-like regions (referred here as P1-P5, respectively) within the chromosome (Fig. 5a ${ }^{42}$. Based on systematic evaluation through individual and combinatory deletion of the prophages, we demonstrated that P5 does not have a gene encoding integrase as an intact phage does and is responsible for the Clostocin O synthesis (Fig. 5b \& c, and Supplementary Fig. 6; Supplementary Note 4, and Supplementary Methods $4-5)^{41}$, and further, we confirmed that P1 represents the lysogenic phage TBP2 genome ${ }^{43}$ (Fig. 5d \& e, and Supplementary Fig. 7). However, interestingly, the phage image we obtained was different from what was described before. The TBP2 phage we observed in this study consists of a head of roughly $50 \mathrm{~nm}$ wide and a tail of about $150 \mathrm{~nm}$ long (Fig. 5e \& Supplementary Fig. 7); while the phage presented in the image by Schüler et al. comprises a 360 $\mathrm{nm}$ long tail as well as a base structure with tail tube and tail fibers $^{43}$. Both $\triangle \mathrm{P} 1234$ (with the deletion of P1-P4) and $\Delta \mathrm{P} 12345$ (with the deletion of P1-P5) exhibited improved cell growth, enhanced butanol production, and prolonged cell integrity during fermentation (Fig. 5f-i, Supplementary Note 4, Supplementary Figs. 8, 9, $10 \& 11$, and Supplementary Method 6). $\Delta \mathrm{P} 12345$ should be a more stable platform as there is no cell lysis at any induction conditions with mitomycin C (Fig. 5d; Supplementary Note 4, Supplementary Figs. 12 \& 13). While $\triangle \mathrm{P} 1234$ showed similar growth and even slightly higher butanol production compared to $\Delta \mathrm{P} 12345$ (Fig. $5 \mathrm{~h} \& \mathrm{i}$ ).

Thus, in a further step, we used $\Delta \mathrm{P} 1234$ and $\Delta \mathrm{P} 12345$ as the platform to be engineered for enhanced and more stable BA production. We deleted Cspa_c47560 and integrated sadh-hydG cluster in both $\Delta \mathrm{P} 1234$ and $\Delta \mathrm{P} 12345$, and obtained FJ-1200 and FJ-1300 correspondingly. The plasmid pMTL- $\mathrm{P}_{\text {adh }}$-atf1-MinD was transformed into FJ-1200 and FJ-1300, generating FJ-1201 and FJ-1301, respectively. Fermentation results showed that
FJ-1201 and FJ-1301 produced $19.7 \mathrm{~g} / \mathrm{L}$ and $19.4 \mathrm{~g} / \mathrm{L}$ BA, respectively, which were both higher than FJ-308 (Fig. 6a \& Supplementary Table 1). BA production in both FJ-1201 and FJ1301 could be completed within $48 \mathrm{~h}$, resulting in a productivity of $\sim 0.41 \mathrm{~g} / \mathrm{L} / \mathrm{h}$, which was significantly higher than $0.23 \mathrm{~g} / \mathrm{L} / \mathrm{h}$ in FJ-308. BA yield in FJ-1201 reached $0.26 \mathrm{~g} / \mathrm{g}$, which was also higher than FJ-308 $(0.24 \mathrm{~g} / \mathrm{g})$. We further determined BA concentrations in the fermentation broth as $0.6 \mathrm{~g} / \mathrm{L}$ and $0.5 \mathrm{~g} / \mathrm{L}$ respectively for the fermentation with FJ-1201 and FJ-1301. Taken together, total BA production in FJ-1201 was $20.3 \mathrm{~g} / \mathrm{L}$. It is 2400 -fold higher than that has been previously reported ${ }^{25}$, and also 14.8 -fold higher than that by the very recently reported $C$. diolis strain 27 .

Besides BA production, BB production in FJ-1201 also reached $0.9 \mathrm{~g} / \mathrm{L}$, which was significantly higher than in FJ-308 $(0.01 \mathrm{~g} / \mathrm{L})$ and in C. pasteurianum J-5 $(0.3 \mathrm{~g} / \mathrm{L})$ (Fig. 2b, Fig. 6a, \& Supplementary Table 1$)$. This level $(0.9 \mathrm{~g} / \mathrm{L})$ was 18.6 -fold higher than the BB production that has been previously reported $(0.05 \mathrm{~g} / \mathrm{L}$ in C. acetobutylicum $)^{25}$. All these results confirmed our hypothesis that the elimination of prophages would make more robust host strains for enhanced and more stable ester production.

Expressing SAAT in FJ-1200 further enhanced BB production. As demonstrated in Fig. 2b, SAAT and EHT1 were more relevant for $\mathrm{BB}$ production. Therefore, to achieve higher $\mathrm{BB}$ production, we expressed saat and eht1 in FJ-1200 and obtained FJ-1202 and FJ-1203, respectively. Fermentation showed that FJ-1202 and FJ-1203 produced 1.3 and $0.2 \mathrm{~g} / \mathrm{L} \mathrm{BB}$, respectively (Fig. 6d). Further, we added MinD C-tag to the SAAT and introduced the recombinant gene into FJ-1200 and obtained FJ-1204 for an attempt to further improve $\mathrm{BB}$ production as observed for BA production in FJ-308. However, BB production in FJ-1204 was only $1.0 \mathrm{~g} / \mathrm{L}$. Notwithstanding, $1.3 \mathrm{~g} / \mathrm{L} \mathrm{BB}$ obtained in FJ-1202 is 25.8-fold higher than the level that has been previously reported ${ }^{25}$.

Ester production with biomass hydrolysates as substrate. Fermentations were carried out using biomass hydrolysates as the substrate. In the hydrolysates, besides sugars $(57.4 \mathrm{~g} / \mathrm{L}$ glucose and $27.2 \mathrm{~g} / \mathrm{L}$ xylose) as carbon source, there were also nutrients converted from biomass (corn stover). Therefore, we tested the effect of organic nitrogen (yeast extract and tryptone) of various levels on ester production. Interestingly, results showed that the BA production of $17.5 \mathrm{~g} / \mathrm{L}$ was achieved in FJ-1201 (in the extractant phase) without any exogenous nitrogen source supplemented (Supplementary Table 2). In addition, $0.3 \mathrm{~g} / \mathrm{L} \mathrm{BA}$ was detected in aqueous phase, making a total BA production of $17.8 \mathrm{~g} / \mathrm{L}$ in FJ-1201 (Fig. 6a \& Supplementary Table 2). Although this was slightly lower than when glucose was used as substrate $(20.3 \mathrm{~g} / \mathrm{L})$, fermentation with hydrolysates did not need any supplementation of nutrients, which could significantly reduce production cost. We further performed fermentation in $500 \mathrm{~mL}$ bioreactor with $\mathrm{pH}$ controlled $>5.0$. BA production reached $16.0 \mathrm{~g} / \mathrm{L}$ with hydrolysates and $18.0 \mathrm{~g} / \mathrm{L}$ with glucose as substrate, both of which were lower than results from fermentation under the same conditions but with serum bottles (Fig. $6 \mathrm{~b} \& \mathrm{c}$ ). The lower levels of BA production in larger scale bioreactors (compared to that in serum bottles) could be attributed to various reasons, including but not limited to the architecture of the reactor, the agitation (both mode and speed), and the $\mathrm{pH}$ control. More systematic optimization of the fermentation in the bioreactor needs to be carried out to further enhance BA production at larger scales.

Furthermore, we performed fermentation with FJ-1202 for BB production using hydrolysates in both serum bottles and $500 \mathrm{~mL}$ 
a

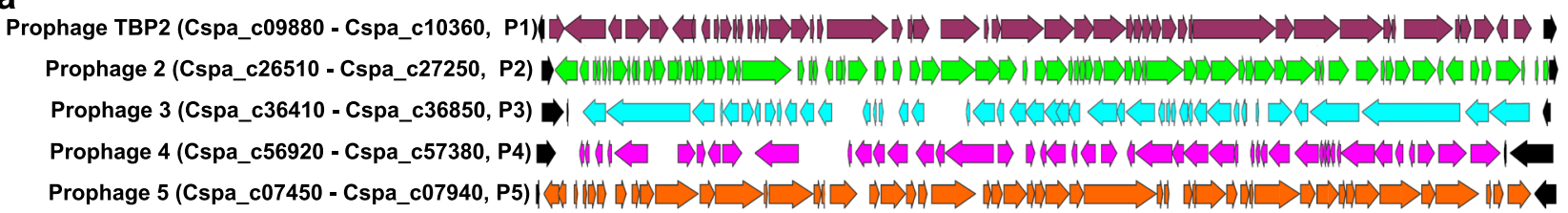

b

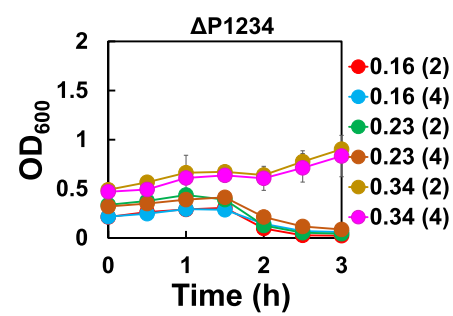

f

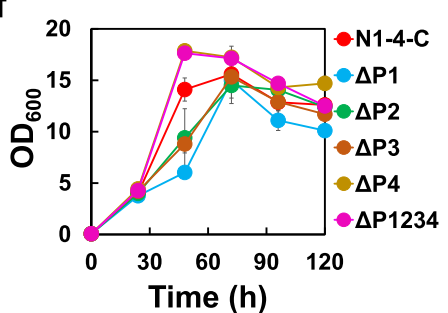

c
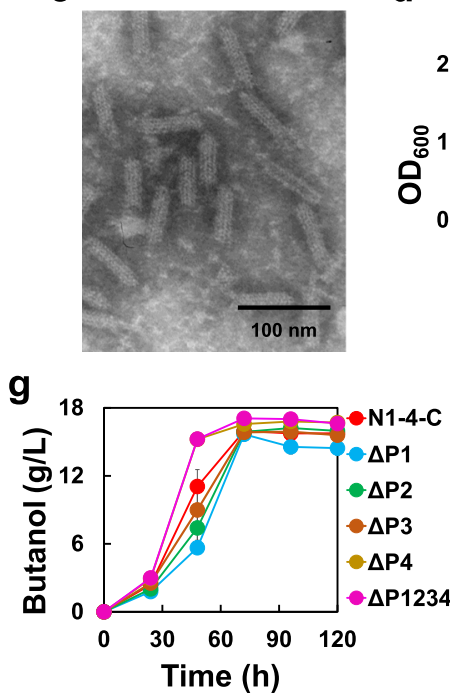

d

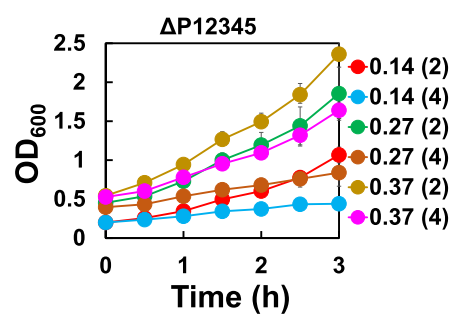

h

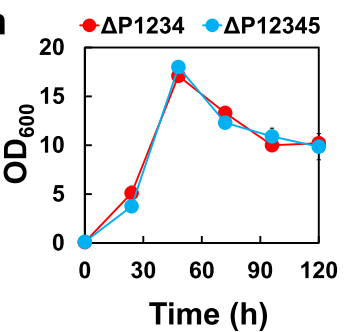

e

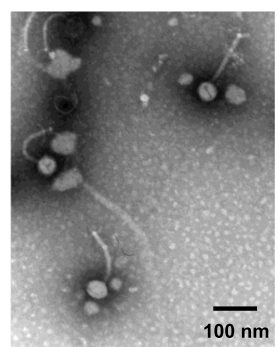

i

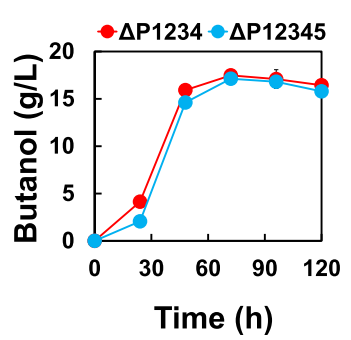

Fig. 5 The deletion of the prophage genomes. a The gene cluster organization of the TBP2 prophage (P1) and other four putative prophages (P2-P5). $\mathbf{b}$ Cell growth profiles of $\triangle \mathrm{P} 1234$ with the induction (at various $\mathrm{OD}_{600}$ ) using mitomycin $\mathrm{C}$ at 2 or $4 \mu \mathrm{g} / \mathrm{mL}$. The value at the right side of the cell growth profile figure represents the actual $\mathrm{OD}_{600}$ value at which mitomycin $\mathrm{C}$ (with the applied concentration included in the parentheses) was added for the induction. Data are presented as mean values \pm SD $(n=3)$. c Transmission electron microscopy image of Clostocin O (observed in the supernatant of $\Delta \mathrm{P} 1234$ after induction with mitomycin C). Similar results were observed in six independent experiments. $\mathbf{d}$ Cell growth profiles of $\Delta \mathrm{P} 12345$ with the induction (at various $\mathrm{OD}_{600}$ ) using mitomycin $\mathrm{C}$ at 2 or $4 \mu \mathrm{g} / \mathrm{mL}$. The value at the right side of the cell growth profile figure represents the actual $\mathrm{OD}_{600}$ value at which mitomycin C (with the applied concentration included in the parentheses) was added for the induction. Data are presented as mean values \pm SD $(n=3)$. e Transmission electron microscopy image of the phage TBP2 particles (observed in the supernatant of $\triangle \mathrm{P} 2345$ after induction with mitomycin C). Similar results were observed in two independent experiments. $\mathbf{f}$ Comparison of the cell growth of prophage deleted mutants and the control N1-4-C strain. Data are presented as mean values \pm SD $(n=3)$. $\mathbf{g}$ Comparison of the butanol production in prophage deleted mutants and the control N1-4-C strain. Data are presented as mean values $\pm \mathrm{SD}(n=3)$. $\mathbf{h}$ Comparison of the cell growth between $\Delta \mathrm{P} 1234$ and $\Delta \mathrm{P} 12345$. Data are presented as mean values \pm SD $(n=3)$. $\mathbf{i}$ Comparison of butanol production between $\Delta \mathrm{P} 1234$ and $\Delta \mathrm{P} 12345$. Data are presented as mean values \pm SD $(n=3)$. Source data underlying Fig. $5 b-i$ are provided as a Source Data file.

bioreactors. Results demonstrated that BB production in serum bottle from hydrolysates was $0.9 \mathrm{~g} / \mathrm{L}$ (compared to $1.3 \mathrm{~g} / \mathrm{L}$ when glucose used as substrate; Fig. $6 \mathrm{~d}$ ). BB production in bioreactor from hydrolysates reached $0.9 \mathrm{~g} / \mathrm{L}$ compared to $1.6 \mathrm{~g} / \mathrm{L}$ when glucose was used as substrate (Fig. 6e \& f). The results were consistent with the case for BA production that lower-level BB was obtained when hydrolysates (compared to glucose) was used as substrate. However, interestingly, larger scale fermentation with bioreactor produced slightly higher level of $\mathrm{BB}$ than the fermentation under the same conditions with serum bottle, which was different from the case for BA production.

Carbon balance analyses were performed based on fermentation results for the production of $\mathrm{BA}$ in $\mathrm{FJ}-1201$ and production of BB in FJ-1202 (Supplementary Data 1). Carbon recovery rate in percentage was defined as total product carbon divided by total substrate carbon multiplied by $100^{44}$. Results indicated that when glucose was used as the carbon source, the carbon recovery rate for FJ-1201 is $100.5 \%$ in serum bottle fermentation and $101.8 \%$ in bioreactor fermentation, respectively; while the carbon recovery rate for FJ-1202 is $89.4 \%$ in serum bottle fermentation and $98.3 \%$ in bioreactor fermentation, respectively. When biomass hydrolysates were used as the carbon source, the carbon recovery rate for $\mathrm{FJ}-1201$ is $93.1 \%$ in serum bottle fermentation and $95.0 \%$ in bioreactor fermentation, respectively; while the carbon recovery rate for FJ-1202 is $87.8 \%$ in serum bottle fermentation and $95.6 \%$ in bioreactor fermentation, respectively.

Techno-economic analysis for BA production. We performed a techno-economic analysis (TEA) to evaluate the economic competitiveness of BA production from corn stover at a process capacity of 2500 MT wet corn stover (20\% moisture) per day. The whole process was developed based on the previous process using the deacetylation and disk refining (DDR) pretreatment to produce corn stover hydrolysates ${ }^{45}$, which was the substrate used for our fermentation experiments to produce BA. The detailed process information is summarized in Supplementary Method 7. The process is composed of eight sections including feedstock handling, pretreatment and hydrolysis, BA fermentation, product recovery (distillation), wastewater treatment, steam and electricity generation, utilities, and chemical and product storage (Fig. 7a). The detailed material balance, key operation parameters, purchased price of raw materials and utilities and the selling price of coproducts (butanol, IPA, and surplus electricity) were shown in Supplementary Tables 3, $4 \& 5$. The process flow diagram of product recovery for TEA was shown in Supplementary Fig. 14. Figure $7 \mathrm{~b}$ shows the equipment cost distribution of each process 
a

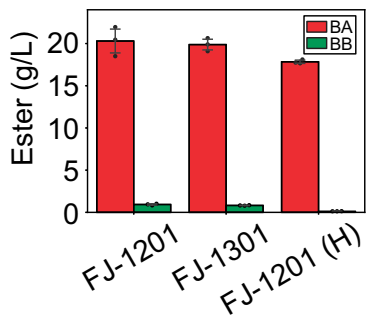

d

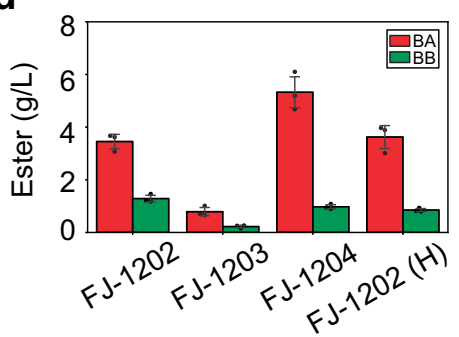

b

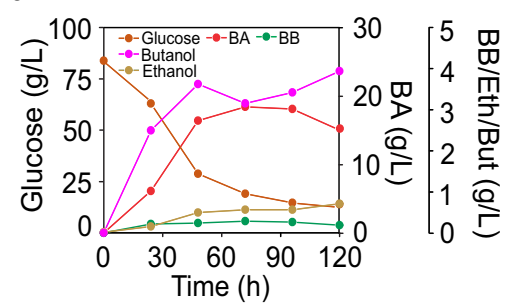

e

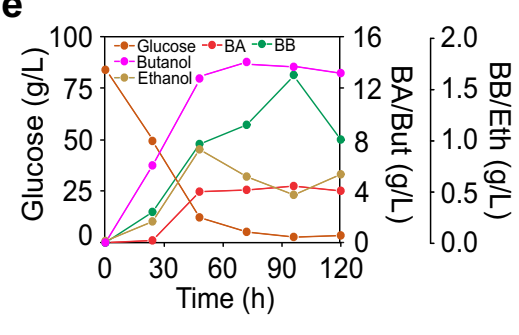

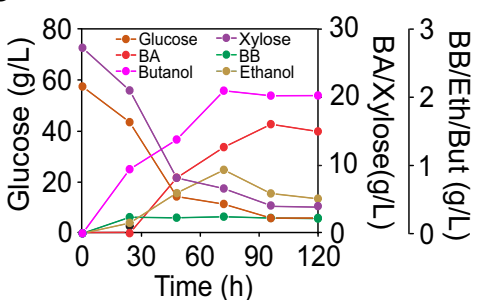

f

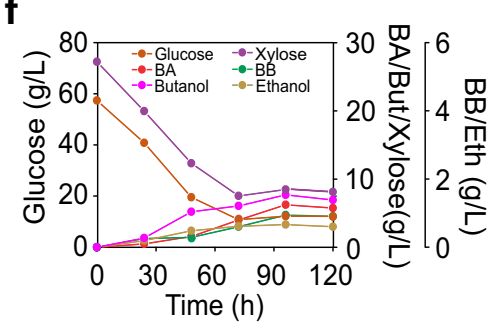

Fig. 6 Fermentation results of the engineered strains for fatty acid ester production. a BA production in serum bottles using glucose (FJ-1201 and FJ1301) or biomass hydrolysates (FJ-1201 (H)) as the substrate. Data are presented as mean values \pm SD $(n=3)$. b BA fermentation kinetics in FJ-1201 in $500 \mathrm{~mL}$ bioreactor using glucose as the substrate. c BA fermentation kinetics in FJ-1201 in $500 \mathrm{~mL}$ bioreactor using biomass hydrolysates as the substrate. d BB production in serum bottles using glucose (FJ-1202, FJ-1203, and FJ-1204) or biomass hydrolysates (FJ-1202 (H)) as the substrate. Data are presented as mean values \pm SD $(n=3)$. e BB fermentation kinetics in FJ-1202 in $500 \mathrm{~mL}$ bioreactor using glucose as the substrate. $\mathbf{f}$ BB fermentation kinetics in FJ-1202 in $500 \mathrm{~mL}$ bioreactor using biomass hydrolysates as the substrate. For fermentations with bioreactors, a single set of fermentation was initially carried out with each of the engineered strains due to the limited availability of biomass hydrolysates. When more biomass hydrolysates became available, the composition (primarily the glucose:xylose ratio) of the new biomass hydrolysates was very different from the previous ones that were used; thus, no further replication of the fermentations with bioreactors was conducted. BA: butyl acetate, BB: butyl butyrate, Eth: ethanol, But: butanol. Source data are provided as a Source Data file.

sections, with a total installed equipment cost of \$263 million. The fermentation, steam \& electricity co-generation, and wastewater treatment contribute significant percentage to the total installed equipment cost, which aligns well with previous TEA models for chemical production from biomass via fermentation ${ }^{46,47}$. It is worth noting that the high installed cost for the wastewater treatment (\$59.8 million, Supplementary Table 6) is mainly attributed to the high costs of major equipment, including anaerobic digester, aeration digester, and membrane reactors, used to treat wastewater into clean water that can be recycled back to the system (see details about the process description in Supplementary Method 7). Previous studies on the lignocellulosic biofuel process with a similar capacity showed that the installed cost of wastewater treatment facility was about $\$ 50$ million $^{45,46,48}$, which is very close to the estimated value in this study. The total capital investment is $\$ 472$ million by taking consideration of additional direct cost, indirect cost as well as working capitals (Supplementary Table 6). From the process model, $95.2 \mathrm{~kg}$ of BA can be produced from 1 MT of corn stover, meanwhile significant amounts of butanol $(11.1 \mathrm{~kg})$, isopropanol $(15.5 \mathrm{~kg})$ and surplus electricity $(209 \mathrm{kWh})$ are produced as co-products (Fig. 7c). The BA production cost was estimated to be $\$ 986 /$ MT (Fig. 7 d) with the detailed calculation shown in Supplementary Table 3 . This production cost is much lower than the current BA market price ranging between $\$ 1200$ and $\$ 1400$ per MT in year 2019 (based on the quotes from the industry ${ }^{49}$ ), showing the high economic competitiveness of BA production using our engineered strain. By looking into the cost breakdown, the corn stover feedstock cost contributes the most $(38.2 \%)$ to the BA production cost, followed by other chemicals (22.3\%) and capital deprecation (18.0\%) and utilities (14.5\%). Sensitivity analysis shows that corn stover price, BA yield, and BA titer are the most sensitive input parameters to the BA production cost (Supplementary Fig. 15).

\section{Discussion}

Although tremendous efforts have been invested on biofuel/biochemical research worldwide, very limited success has been achieved. Our central hypothesis is that metabolically engineering of microorganisms for high-value and easy-recoverable bioproduct production (which can lead to high titer and productivity) could be a feasible route to establish highly efficient and economically viable biofuel/biochemical production processes. Here we tested this hypothesis by engineering solventogenic clostridia for efficient ester (high-value and easy-recoverable) production. Our group have previously established versatile genome engineering tools for clostridia ${ }^{15,18,33}$, putting us at a strong position to perform this study.

Based on the systematic screening of host strains and enzymes as well as multiple rounds of rational metabolic engineering: enriching precursors (alcohols and acetyl-CoA) for ester production, dynamically expressing heterologous ester-production pathways, rationally organizing ester-synthesis enzymes, and improving strain robustness by eliminating putative prophages, we ultimately obtained strains for efficient production of esters in both synthetic fermentation medium and biomass hydrolysates (Supplementary Note 5, Supplementary Table 7, and Supplementary Discussion). To the best of our knowledge, the production levels of $\mathrm{BA}$ and $\mathrm{BB}$ we achieved set up the records. Overall, we demonstrate that clostridia are excellent platforms for valuable biofuel and biochemical production. The general principles that we demonstrated herein, including (1) selecting the most appropriate host for targeted bioproduction and (2) engineering the host for producing high value and easily recoverable products, are highly applicable to other relevant bioprocesses, and may result in breakthroughs in biofuel/biochemical production and general bioeconomy. 


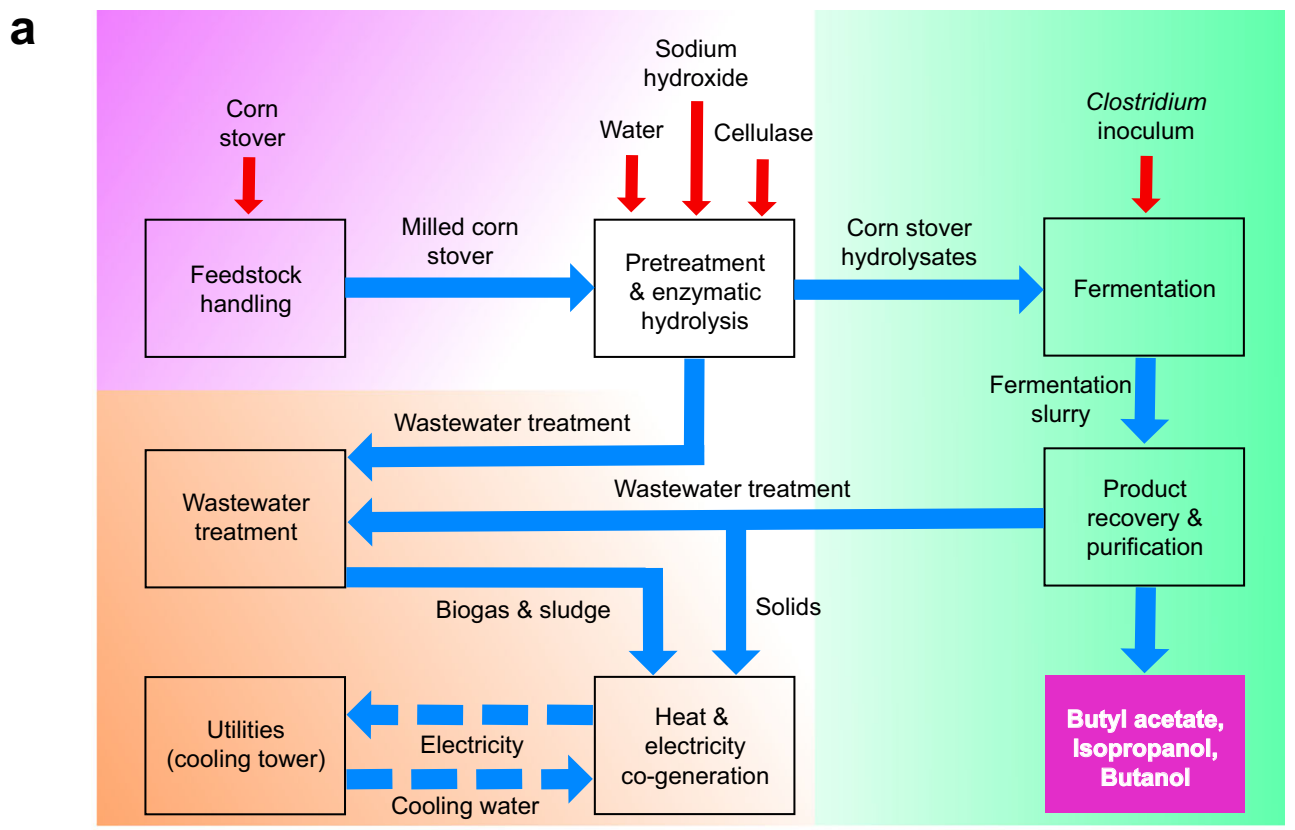

b

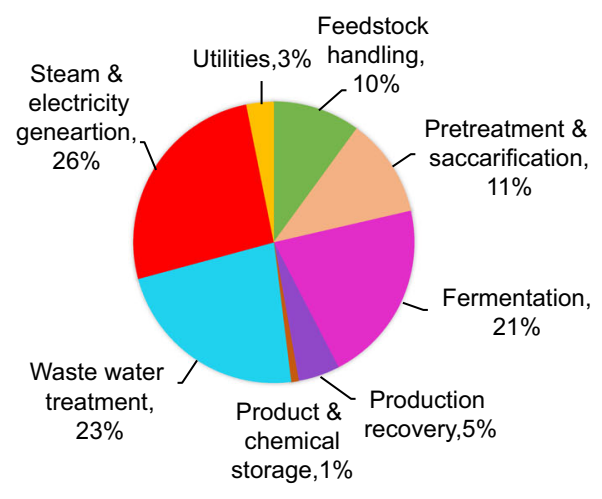

C

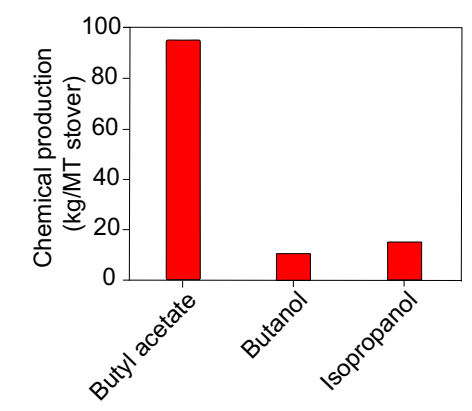

d

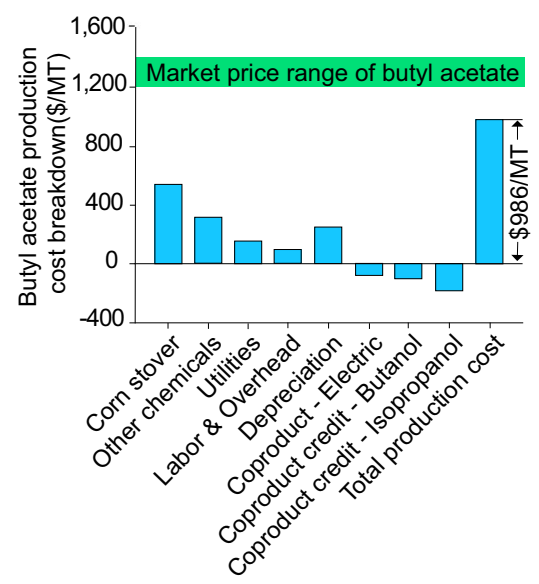

Fig. 7 Techno-economic analysis of butyl acetate production from corn stover. a Process overview. $\mathbf{b}$ Total installed equipment cost. c Chemical production from each metric tonne (MT) of corn stover. $\mathbf{d}$ Butyl acetate production cost. Source data underlying Fig. $7 b-d$ are provided as a Source Data file.

\section{Methods}

Microorganisms and cultivation conditions. All the strains used in this study are listed in Supplementary Data 2. C. pasteurianum ATCC 6013 and C. saccharoperbutylacetonicum N1-4 (HMT) (DSM 14923) were requested from American Type Culture Collection (ATCC) and Deutsche Sammlung von Mikroorganismen und Zellkulturen (DSMZ), respectively. C. beijerinckii NCIMB 8052 was provided by Dr. Hans P. Blaschek ${ }^{18}$. C. tyrobutyricum $\Delta$ cat $1:$ adhE1 and C. tyrobutyricum $\triangle$ cat $1:: a d h E 2$ are hyper-butanol producing mutants constructed in our lab ${ }^{15}$. All the clostridial strains were grown in an anaerobic chamber $\left(\mathrm{N}_{2}-\mathrm{CO}_{2}-\mathrm{H}_{2}\right.$ with a volume ratio of $85: 10: 5)$ at $35^{\circ} \mathrm{C}$. Strains of C. tyrobutyricum, C. saccharoperbutylacetonicum and C. beijerinckii were cultivated using tryptone-glucoseyeast extract (TGY) medium ${ }^{50}$, while strains of $C$. pasteurianum were cultivated using $2 \times$ YTG medium $^{51}$. When required, clarithromycin (Cla) or thiamphenicol (Tm) was supplemented into the medium at a final concentration of $30 \mu \mathrm{g} / \mathrm{mL}$ and $15 \mu \mathrm{g} / \mathrm{mL}$, respectively. E. coli $\mathrm{DH} 5$ a was used for routine plasmid propagation and maintenance. E. coli CA434 was used as the donor strain for plasmid conjugation for C. tyrobutyricum. Strains of E. coli were grown aerobically at $37^{\circ} \mathrm{C}$ in LuriaBertani (LB) medium supplemented with $100 \mu \mathrm{g} / \mathrm{mL}$ ampicillin (Amp), $50 \mu \mathrm{g} / \mathrm{mL}$ kanamycin (Kan) or $34 \mu \mathrm{g} / \mathrm{mL}$ chloramphenical $(\mathrm{Cm})$ as needed.

Plasmid construction. All the plasmids used in this study are listed in Supplementary Data 2, and all the primers used in this study are listed in Supplementary Data 3.
The plasmids pMTL82151 and pTJ1 were used for heterogeneous gene expression ${ }^{50,52}$. The promoter of the cat1 gene $\left(C T K\right.$ C06520) $\left(\mathrm{P}_{\text {cat }}\right)$ and the promoter of the $t h l$ gene $\left(C T K \_C 01450\right)\left(\mathrm{P}_{\text {thl }}\right)$ from C. tyrobutyricum ATCC 25755 were amplified and inserted into pMTL82151 at the EcoRI site, and the generated plasmids were named as pMTL82151- $\mathrm{P}_{c a t}$ and pMTL82151- $\mathrm{P}_{t h l}$, respectively. Promoters of the following gene, pflA (Cspa_c13710) $\left(\mathrm{P}_{p f l}\right)$, ald (Cspa_c56880) $\left(\mathrm{P}_{a l d}\right)$, adh (Cspa_c04380) $\left(\mathrm{P}_{a d h}\right)$ and bdh (Cspa_c56790) $\left(\mathrm{P}_{b d h}\right)$, all from $C$ saccharoperbutylacetonicum N1-4 (HMT) were amplified and inserted into pMTL82151 at the EcoRI site, and the generated plasmids were named as

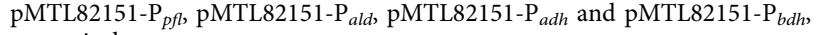
respectively.

The vaat gene from Fragaria vesca, the saat gene from F. ananassa and the atf1 gene from $S$. cerevisiae were amplified from plasmids pDL006, pDL001 and pDL004, respectively ${ }^{2,24}$. The atf1' (the codon optimized atf1 gene), eht1 from S. cerevisiae 22 , and lipaseB from Candida antarctica ${ }^{53}$ were all synthesized by GenScript (Piscataway, NJ, USA). The obtained gene fragments of vaat, saat, atf1, atf1', eth1, and lipaseB were inserted between the BtgZI and EcoRI sites in pMTL82151- $\mathrm{P}_{\text {cat }}$, generating pMTL- $\mathrm{P}_{\text {cat }}$-vaat, pMTL- $\mathrm{P}_{\text {cat }}$-saat, pMTL- $\mathrm{P}_{\text {cat }}$-atf1, pMTL- $\mathrm{P}_{\text {cat }}-$ atf1', pMTL- $\mathrm{P}_{\text {cat }}-$ eht 1 , and pMTL- $\mathrm{P}_{\text {cat }}$-lipaseB, respectively. The atf 1 gene was inserted between the BtgZI and EcoRI sites in pMTL82151- $\mathrm{P}_{t h l}$, generating pMTL- $\mathrm{P}_{t h l^{-}}$atfl.

The $\mathrm{P}_{\text {cat }}$ promoter and the gene fragments of vaat, saat, atf1, eth1, and lipase

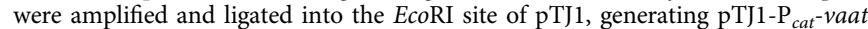

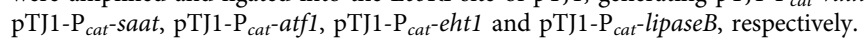


The atf1 gene was inserted into the EcoRI site of pMTL82151-P $\mathrm{P}_{p f}$, pMTL82151$\mathrm{P}_{\text {ald }}$, pMTL82151- $\mathrm{P}_{a d h}$ and pMTL82151- $\mathrm{P}_{b d h}$, generating pMTL- $\mathrm{P}_{p f l}-$ atf1, $\mathrm{pMTL}-$ $\mathrm{P}_{\text {ald }}$-atf 1 , pMTL- $\mathrm{P}_{\text {adh }}$-atf1 and pMTL- $\mathrm{P}_{b d h}$-atf1, respectively.

DNA fragments of CC-Di-A, CC-Di-B, MinD and $p d u A^{*}$ were synthesized by GenScript (Piscataway, NJ, USA). The MinD-tag was fused to the end of atf1 with PCR and ligated into the EcoRI site of pMTL82151-P ${ }_{a d h}$, generating pMTL$\mathrm{P}_{\text {adh }}$-atf1-MinD. In addition, the MinD-tag was fused to the end of saat with PCR and inserted between the BtgZI and EcoRI sites of pMTL82151- $\mathrm{P}_{c a t}$, generating pMTL-P cat $_{\text {-saat-MinD. }}$.

The synthesized CC-Di-A fragment was ligated into the EcoRI site of pMTL82151-P $\mathrm{P}_{a d h}$, generating pMTL-P $\mathrm{P}_{a d h}$-CC-Di-A. The DNA fragments of atf1 and atf1-MinD were amplified from pMTL-P ${ }_{\text {adh }}-$ atf1 and pMTL-P ${ }_{\text {adh }}$-atf1-MinD and then inserted into the EcoRI site of pMTL-P ${ }_{a d h}$-CC-Di-A, obtaining pMTL$\mathrm{P}_{a d h}$-A-atf1 and pMTL-P $\mathrm{P}_{a d h}$-A-atf1-MinD. The CC-Di-B sequence with the nifJ gene and $C C-D i-B$ with the $b d h A$ gene were subsequently inserted into the $K p n I$ site of pMTL-P $\mathrm{P}_{a d h}-\mathrm{A}$-atf 1 , generating pMTL- $\mathrm{P}_{a d h}-\mathrm{A}-a t f 1-\mathrm{B}-n i f J$ and pMTL-P $\mathrm{P}_{a d h}-\mathrm{A}-$ atfl-B-nifJ-B-bdhA. The DNA fragments of CC-Di-B-pduA*, CC-Di-A-niff, CC-Di$A-b d h A$ were inserted into the EcoRI site of pTJ1- $\mathrm{P}_{c a t}$ generating pTJ1- $\mathrm{P}_{c a t}-\mathrm{B}$ $p d u A^{*}, \mathrm{pTJ1}-\mathrm{P}_{c a t}-\mathrm{B}-p d u A^{*}-\mathrm{A}-n i f J$ and $\mathrm{pTJ1}-\mathrm{P}_{c a t}-\mathrm{B}-p d u A^{*}-\mathrm{A}-n i f J-\mathrm{A}-b d h A$, respectively.

For the gene deletion or integration in C. saccharoperbutylacetonicum, all the relevant plasmids were constructed based on pYW34, which carries the customized CRISPR-Cas9 system for genome editing in C. saccharoperbutylacetonicum ${ }^{18,33}$. The promoter $\mathrm{P}_{\mathrm{J} 23119}$ and the gRNA (with 20-nt guide sequence targeting on the specific gene) were amplified by two rounds of PCR with primers N-20nt/YW1342 and YW1339/YW1342 (N represents the targeted gene) ${ }^{33}$. The obtained fragment was then inserted into pYW34 (digested with BtgZI and NotI) through Gibson Assembly, generating the intermediate vectors. For gene deletion, the fragment containing the two corresponding homology arms $(\sim 500 \mathrm{bp}$ for regular gene deletion; $1000-2000 \mathrm{bp}$ for prophage deletion) for deleting the specific gene through homologous recombination was amplified and inserted into the NotI site of the obtained intermediate vector as described above, generating pYW34- $\Delta \mathrm{N}(\mathrm{N}$ represents the targeted gene) (Supplementary Fig. 16). For gene integration, the fragment containing the two corresponding homology arms ( 1000 bp for each), the promoter and the gene fragment to be integrated, was amplified and inserted into the NotI site of the obtained intermediate vector as described above, generating the final plasmid for gene integration purpose. The details about plasmid transformation and mutant verification have been described in Supplementary Method 8.

Fermentation with glucose or glycerol as the substrate. For the fermentation for ester production, the C. pasteurianum strain was cultivated in Biebl medium ${ }^{54}$ with $50 \mathrm{~g} / \mathrm{L}$ glycerol as the carbon source at $35^{\circ} \mathrm{C}$ in the anaerobic chamber. When the $\mathrm{OD}_{60}$ reached $\sim 0.8$, the seed culture was inoculated at a ratio of $10 \%$ into $100 \mathrm{~mL}$ of the same medium in a $250 \mathrm{~mL}$ serum bottle and then cultivated at an agitation of $150 \mathrm{rpm}$ and $30^{\circ} \mathrm{C}$ (on a shaker incubator) for $72 \mathrm{~h}$. The C. beijerinckii strain was cultivated in TGY medium until the $\mathrm{OD}_{600}$ reached $\sim 0.8$. Then the seed culture was inoculated at a ratio of $5 \%$ into $100 \mathrm{~mL}$ P2 medium along with $60 \mathrm{~g} / \mathrm{L}$ glucose and $1 \mathrm{~g} / \mathrm{L}$ yeast extract in a $250 \mathrm{~mL}$ serum bottle. The fermentation was carried out at an agitation of $150 \mathrm{rpm}$ and $37^{\circ} \mathrm{C}$ for $72 \mathrm{~h}^{50}$. The C. tyrobutyricum strain was cultivated in RCM medium at $35^{\circ} \mathrm{C}$ until the $\mathrm{OD}_{600}$ reached $\sim 1.5$. Then the seed culture was inoculated at a ratio of $5 \%$ into $200 \mathrm{~mL}$ fermentation medium (containing: $50 \mathrm{~g} / \mathrm{L}$ glucose, $5 \mathrm{~g} / \mathrm{L}$ yeast extract, $5 \mathrm{~g} / \mathrm{L}$ tryptone, $3 \mathrm{~g} / \mathrm{L}$ $\left(\mathrm{NH}_{4}\right)_{2} \mathrm{SO}_{4}, 1.5 \mathrm{~g} / \mathrm{L} \mathrm{K}_{2} \mathrm{HPO}_{4}, 0.6 \mathrm{~g} / \mathrm{L} \mathrm{MgSO}{ }_{4} \cdot 7 \mathrm{H}_{2} \mathrm{O}, 0.03 \mathrm{~g} / \mathrm{L} \mathrm{FeSO}{ }_{4} \cdot 7 \mathrm{H}_{2} \mathrm{O}$, and $1 \mathrm{~g} / \mathrm{L}$ L-cysteine) in a $500 \mathrm{~mL}$ bioreactor (GS-MFC, Shanghai Gu Xin biological technology Co., Shanghai, China) and the fermentation was carried out at an agitation of $150 \mathrm{rpm}$ and $37^{\circ} \mathrm{C}$ for $120 \mathrm{~h}$ with $\mathrm{pH}$ controlled $>6.0^{9}$. The C. saccharoperbutylacetonicum strain was cultivated in TGY medium at $35^{\circ} \mathrm{C}$ in the anaerobic chamber until the $\mathrm{OD}_{600}$ reached $\sim 0.8$. Then the seed culture was inoculated at a ratio of $5 \%$ into $100 \mathrm{~mL}$ P2 medium along with $80 \mathrm{~g} / \mathrm{L}$ glucose and $6 \mathrm{~g} / \mathrm{L}$ tryptone, and $2 \mathrm{~g} / \mathrm{L}$ yeast extract in a $250 \mathrm{~mL}$ serum bottle ${ }^{17}$. The fermentation was carried out at an agitation of $150 \mathrm{rpm}$ and $30^{\circ} \mathrm{C}$ for $120 \mathrm{~h}^{50}$. For the fermentation at larger scales in bioreactors, it was carried out in a $500 \mathrm{~mL}$ fermenter (GS-MFC, Shanghai Gu Xin biological technology Co., Shanghai, China) with a working volume of $250 \mathrm{~mL}$ with $\mathrm{pH}$ controlled $>5.0$, at $50 \mathrm{rpm}$ and $30^{\circ} \mathrm{C}$ for $120 \mathrm{~h}$. Samples were taken every $24 \mathrm{~h}$ for analysis.

For all fermentations in the serum bottle, a needle and hosepipe were connected to the top of bottle for releasing the gases produced during the fermentation. For all the fermentations for ester production, the extractant $n$-hexadecane was added into the fermentation with a ratio of 1:1 (volume of the extractant vs. volume of fermentation broth) for in situ ester extraction. The reported ester concentrations were the determined values in the extractant phase.

Fermentation with biomass hydrolysates as the substrate. The biomass hydrolysates was kindly provided by Dr. Daniel Schell from National Renewable Energy Laboratory (NREL), which was generated from corn stover through the innovative deacetylation and mechanical refining in a disc refiner (DDR) approach $^{55}$. For the fermentation, the biomass hydrolysate was diluted and supplemented into the P2 medium as the carbon source (with final sugar concentrations of $57.4 \mathrm{~g} / \mathrm{L}$ glucose and $27.2 \mathrm{~g} / \mathrm{L}$ xylose). In addition, various concentrations of yeast extract $(\mathrm{Y}, \mathrm{g} / \mathrm{L})$ and tryptone $(\mathrm{T}, \mathrm{g} / \mathrm{L})$ were also added as the nitrogen source to evaluate their effects on the fermentation performance: $0 \mathrm{Y}+0 \mathrm{~T} ; 1 \mathrm{Y}+3 \mathrm{~T}$ and $2 \mathrm{Y}+6 \mathrm{~T}$. The fermentation was carried out under the same conditions as described above at $100 \mathrm{~mL}$ working volume in a $250 \mathrm{~mL}$ serum bottle.

Analytical methods. Concentrations of acetone, ethanol, butanol, isopropanol, acetic acid, butyric acid and glucose were measured using a high-performance liquid chromatography (HPLC, Agilent Technologies 1260 Infinity series, Santa Clara, CA) with a refractive index Detector (RID), equipped with an Aminex HPX$87 \mathrm{H}$ column (Bio-Rad Laboratories, Hercules, CA). The column was eluted with $5 \mathrm{mM} \mathrm{H}_{2} \mathrm{SO}_{4}$ at a flow rate of $0.6 \mathrm{~mL} / \mathrm{min}$ at $25^{\circ} \mathrm{C}$. The concentration of the ester in the $n$-hexadecane phase was quantified using a gas chromatography-mass spectrometry (GC-MS, Agilent Technologies 6890N, Santa Clara, CA) equipped with an HP- 5 column $(60 \mathrm{~m} \times 0.25 \mathrm{~mm}, 0.25 \mathrm{~mm}$ film thickness). Helium was used as the carrier gas. The initial temperature of the oven was set at $30^{\circ} \mathrm{C}$ for $2 \mathrm{~min}$, followed by a ramp of $10{ }^{\circ} \mathrm{C} / \mathrm{min}$ to reach $300^{\circ} \mathrm{C}$, and a ramp of $2{ }^{\circ} \mathrm{C} / \mathrm{min}$ to reach the final temperature of $320^{\circ} \mathrm{C}$, which was then held for $2 \mathrm{~min}$. The detector was kept at $225^{\circ} \mathrm{C}^{9}$.

All fermentation data were analyzed using Microsoft Excel 2016 (Microsoft Corporation, WA). Origin 2016 (OriginLab Corporation, MA) and Python 3.7 (Python Software Foundation, DE) were used for statistical analysis.

Reporting summary. Further information on research design is available in the Nature Research Reporting Summary linked to this article.

\section{Data availability}

Data supporting the findings of this work are available within the paper and its Supplementary Information files. A reporting summary for this article is available as a Supplementary Information file. Source data are provided with this paper.

Received: 13 April 2020; Accepted: 26 May 2021;

Published online: 16 July 2021

\section{References}

1. Stephanopoulos, G. Challenges in engineering microbes for biofuels production. Science 315, 801-804 (2007).

2. Layton, D. S. \& Trinh, C. T. Expanding the modular ester fermentative pathways for combinatorial biosynthesis of esters from volatile organic acids. Biotechnol. Bioeng. 113, 1764-1776 (2016).

3. Park, Y. C., Shaffer, C. E. H. \& Bennett, G. N. Microbial formation of esters. Appl. Microbiol. Biotechnol. 85, 13-25 (2009).

4. U.S. esters market size, share \& trends analysis report by product (fatty esters, phosphate esters, acrylic esters, cellulose esters, allyl and aromatic esters), by application, and segment forecasts, 2019-2025 (https://www. grandviewresearch.com/industry-analysis/us-esters-market). Grand view reserach (2019).

5. Rodriguez, G. M., Tashiro, Y. \& Atsumi, S. Expanding ester biosynthesis in Escherichia coli. Nat. Chem. Biol. 10, 259-265 (2014).

6. Liu, Y., Lotero, E. \& Goodwin, J. G. Effect of water on sulfuric acid catalyzed esterification. J. Mol. Catal. A: Chem. 245, 132-140 (2006).

7. van den Berg, C., Heeres, A. S., van der Wielen, L. A. \& Straathof, A. J. Simultaneous clostridial fermentation, lipase-catalyzed esterification, and ester extraction to enrich diesel with butyl butyrate. Biotechnol. Bioeng. 110, 137-142 (2013).

8. Stergiou, P.-Y. et al. Advances in lipase-catalyzed esterification reactions Biotechnol. Adv. 31, 1846-1859 (2013)

9. Zhang, Z. T., Taylor, S. \& Wang, Y. In situ esterification and extractive fermentation for butyl butyrate production with Clostridium tyrobutyricum. Biotechnol. Bioeng. 114, 1428-1437 (2017).

10. Loser, C., Urit, T., Keil, P. \& Bley, T. Studies on the mechanism of synthesis of ethyl acetate in Kluyveromyces marxianus DSM 5422. Appl. Microbiol. Biotechnol. 99, 1131-1144 (2015).

11. Verstrepen, K. J. et al. Expression levels of the yeast alcohol acetyltransferase genes ATF1, Lg-ATF1, and ATF2 control the formation of a broad range of volatile esters. Appl. Environ. Microbiol. 69, 5228-5237 (2003).

12. Horton, C. E., Huang, K. X., Bennett, G. N. \& Rudolph, F. B. Heterologous expression of the Saccharomyces cerevisiae alcohol acetyltransferase genes in Clostridium acetobutylicum and Escherichia coli for the production of isoamyl acetate. J. Ind. Microbiol. Biotechnol. 30, 427-432 (2003).

13. Horton, C. E. \& Bennett, G. N. Ester production in E. coli and C. acetobutylicum. Enzym. Microb. Technol. 38, 937-943 (2006).

14. Bohnenkamp, A. C. et al. Multilevel optimisation of anaerobic ethyl acetate production in engineered Escherichia coli. Biotechnol. Biofuels 13, 65 (2020). 
15. Zhang, J., Zong, W., Hong, W., Zhang, Z.-T. \& Wang, Y. Exploiting endogenous CRISPR-Cas system for multiplex genome editing in Clostridium tyrobutyricum and engineer the strain for high-level butanol production. Metab. Eng. 47, 49-59 (2018).

16. Pyne, M. E., Bruder, M. R., Moo-Young, M., Chung, D. A. \& Chou, C. P. Harnessing heterologous and endogenous CRISPR-Cas machineries for efficient markerless genome editing in Clostridium. Sci. Rep. 6, 25666 (2016).

17. Gu, Y. et al. Curing the endogenous megaplasmid in Clostridium saccharoperbutylacetonicum N1-4 (HMT) using CRISPR-Cas9 and preliminary investigation of the role of the plasmid for the strain metabolism. Fuel 236, 1559-1566 (2019).

18. Wang, Y. et al. Bacterial genome editing with CRISPR-Cas9: deletion, Integration, single nucleotide modification, and desirable "clean" mutant selection in Clostridium beijerinckii as an example. ACS Synth. Biol. 5, 721-732 (2016).

19. Yoo, M., Croux, C., Meynial-Salles, I. \& Soucaille, P. Elucidation of the roles of adhE1 and adhE2 in the primary metabolism of Clostridium acetobutylicum by combining in-frame gene deletion and a quantitative system-scale approach. Biotechnol. Biofuels 9, 92 (2016).

20. Han, S.-Y. et al. Highly efficient synthesis of ethyl hexanoate catalyzed by CALB-displaying Saccharomyces cerevisiae whole-cells in non-aqueous phase. J. Mol. Catal. B: Enzymatic 59, 168-172 (2009).

21. Aharoni, A. et al. Identification of the SAAT gene involved in strawberry flavor biogenesis by use of DNA microarrays. Plant Cell 12, 647-661 (2000).

22. Saerens, S. M. et al. The Saccharomyces cerevisiae EHT1 and EEB1 genes encode novel enzymes with medium-chain fatty acid ethyl ester synthesis and hydrolysis capacity. J. Biol. Chem. 281, 4446-4456 (2006).

23. Tai, Y.-S., Xiong, M. \& Zhang, K. Engineered biosynthesis of medium-chain esters in Escherichia coli. Metab. Eng. 27, 20-28 (2015).

24. Layton, D. S. \& Trinh, C. T. Engineering modular ester fermentative pathways in Escherichia coli. Metab. Eng. 26, 77-88 (2014).

25. Noh, H. J., Woo, J. E., Lee, S. Y. \& Jang, Y.-S. Metabolic engineering of Clostridium acetobutylicum for the production of butyl butyrate. Appl. Microbiol. Biotechnol. 102, 8319-8327 (2018).

26. Alonso-Gutierrez, J. et al. Metabolic engineering of Escherichia coli for limonene and perillyl alcohol production. Metab. Eng. 19, 33-41 (2013).

27. Li, A. et al. Developing Clostridium diolis as a biorefinery chassis by genetic manipulation. Bioresour. Technol. 305, 123066 (2020).

28. Nguyen, N. P., Raynaud, C., Meynial-Salles, I. \& Soucaille, P. Reviving the Weizmann process for commercial n-butanol production. Nat. Commun. 9, 3682 (2018).

29. Amador-Noguez, D., Brasg, I. A., Feng, X.-J., Roquet, N. \& Rabinowitz, J. D. Metabolome remodeling during the acidogenic-solventogenic transition in Clostridium acetobutylicum. Appl. Environ. Microbiol. 77, 7984-7997 (2011).

30. Jang, Y. S. et al. Enhanced butanol production obtained by reinforcing the direct butanol-forming route in Clostridium acetobutylicum. mBio 3, e00314 (2012).

31. Wang, S., Huang, H., Moll, J. \& Thauer, R. K. NADP+ reduction with reduced ferredoxin and NADP+ reduction with NADH are coupled via an electronbifurcating enzyme complex in Clostridium kluyveri. J. Bacteriol. 192, 5115-5123 (2010).

32. Liang, J., Huang, H. \& Wang, S. Distribution, evolution, catalytic mechanism, and physiological functions of the flavin-based electron-bifurcating NADH-dependent reduced ferredoxin: NADP(+) oxidoreductase. Front. Microbiol. 10, 373 (2019).

33. Wang, S., Dong, S., Wang, P., Tao, Y. \& Wang, Y. Genome editing in Clostridium saccharoperbutylacetonicum N1-4 with the CRISPR-Cas9 system. Appl. Environ. Microbiol. 83, e00233-00217 (2017).

34. Wang, P., Feng, J., Guo, L., Fasina, O. \& Wang, Y. Engineering Clostridium saccharoperbutylacetonicum for high level Isopropanol-Butanol-Ethanol (IBE) production from acetic acid pretreated switchgrass using the CRISPR-Cas9 system. ACS Sustain. Chem. Eng. 7, 18153-18164 (2019).

35. Lee, J. et al. Metabolic engineering of Clostridium acetobutylicum ATCC 824 for isopropanol-butanol-ethanol fermentation. Appl. Environ. Microbiol. 78, 1416-1423 (2012).

36. Jang, Y. S. et al. Metabolic engineering of Clostridium acetobutylicum for the enhanced production of isopropanol-butanol-ethanol fuel mixture. Biotechnol. Prog. 29, 1083-1088 (2013).

37. Kosaka, T., Nakayama, S., Nakaya, K., Yoshino, S. \& Furukawa, K. Characterization of the sol operon in butanol-hyperproducing Clostridium saccharoperbutylacetonicum strain N1-4 and its degeneration mechanism. Biosci. Biotechnol. Biochem. 71, 58-68 (2007).

38. Lee, M. J. et al. Engineered synthetic scaffolds for organizing proteins within the bacterial cytoplasm. Nat. Chem. Biol. 14, 142-147 (2017).

39. Szeto, T. H., Rowland, S. L., Habrukowich, C. L. \& King, G. F. The MinD membrane targeting sequence is a transplantable lipid-binding helix. J. Biol. Chem. 278, 40050-40056 (2003).

40. Hongo, M., Murata, A. \& Ogata, S. Bacteriophages of Clostridium saccharoperbutylacetonicum: Part XVI. Isolation and some characters of a temperate phage. Agric. Biol. Chem. 33, 337-342 (1969).
41. Ogata, S., Mihara, O., Ikeda, Y. \& Hongo, M. Inducible phage tail-like particles of Clostridium saccharoperbutylacetonicum and its related strains. Agric. Biol. Chem. 36, 1413-1421 (1972).

42. Zhou, Y., Liang, Y., Lynch, K. H., Dennis, J. J. \& Wishart, D. S. PHAST: a fast phage search tool. Nucleic Acids Res. 39, W347-W352 (2011).

43. Schuler, M. A., Stegmann, B. A., Poehlein, A., Daniel, R. \& Durre, P. Genome sequence analysis of the temperate bacteriophage TBP2 of the solvent producer Clostridium saccharoperbutylacetonicum N1-4 (HMT, ATCC 27021). FEMS Microbiol. Lett. 367, fnaa103 (2020).

44. Joseph Formanek, R. M. \& Hans, P. Blaschek Enhanced butanol production by Clostridium beijerinckii BA101 grown in semidefined P2 medium containing 6 percent maltodextrin or glucose. Appl. Environ. Microbiol. 63, 2306-2310 (1997).

45. Humbird, D. et al. Process design and economics for biochemical conversion of lignocellulosic biomass to ethanol:dilute-acid pretreatment and enzymatic hydrolysis of corn stover. (No. NREL/TP-5100-47764). National Renewable Energy Lab.(NREL), Golden, CO (United States) (NREL, 2011).

46. Tao, L. et al. Techno-economic analysis and life-cycle assessment of cellulosic isobutanol and comparison with cellulosic ethanol and n-butanol. Biofuels Bioprod. Bioref. 8, 30-48 (2014).

47. Huang, H., Long, S. \& Singh, V. Techno-economic analysis of biodiesel and ethanol co-production from lipid-producing sugarcane. Biofuels Bioprod. Bioref. 10, 299-315 (2016).

48. Li, W., Ghosh, A., Bbosa, D., Brown, R. \& Wright, M. M. Comparative Techno-economic, uncertainty and life cycle analysis of lignocellulosic biomass solvent liquefaction and sugar fermentation to ethanol. ACS Sustain. Chem. Eng. 6, 16515-16524 (2018).

49. https://www.alibaba.com/product-detail/Purity-99-5-Butyl-Acetate-Cas_62416155 030.html?spm=a2700.galleryofferlist.0.0.67372e613qq3zW\&s=p.

50. Wang, Y. et al. Development of a gene knockout system using mobile group II introns (Targetron) and genetic disruption of acid production pathways in Clostridium beijerinckii. Appl. Environ. Microbiol. 79, 5853-5863 (2013).

51. Schwarz, K. M. et al. Towards improved butanol production through targeted genetic modification of Clostridium pasteurianum. Metab. Eng. 40, 124-137 (2017).

52. Heap, J. T., Pennington, O. J., Cartman, S. T. \& Minton, N. P. A modular system for Clostridium shuttle plasmids. J. Microbiol. Methods 78, 79-85 (2009).

53. Tamalampudi, S. et al. Development of recombinant Aspergillus oryzae wholecell biocatalyst expressing lipase-encoding gene from Candida antarctica. Appl. Microbiol. Biotechnol. 75, 387-395 (2007).

54. Biebl, H. Fermentation of glycerol by Clostridium pasteurianum-batch and continuous culture studies. J. Ind. Microbiol. Biotechnol. 27, 18-26 (2001).

55. Chen, X. et al. A highly efficient dilute alkali deacetylation and mechanical (disc) refining process for the conversion of renewable biomass to lower cost sugars. Biotechnol. Biofuels 7, 98 (2014).

\section{Acknowledgements}

This work was supported by the US Department of Energy's Office of Energy Efficiency and Renewable Energy under Award DE-EE0008483 (Co-Optima), the Agriculture and Food Research Initiative Competitive Grant no. 2018-67021-27715 from the USDA National Institute of Food and Agriculture (NIFA), the Auburn University Intramural Grants Program (IGP), the USDA-NIFA Hatch project (ALA014-1017025), and the Alabama Agricultural Experiment Station. We thank Dr. Hans Blaschek (University of Illinois at Urbana-Champaign) for providing plasmids pYW34 and pTJ1, Dr. Nigel Minton (University of Nottingham, UK) for providing pMTL series of plasmids, Dr. Cong T Trinh (University of Tennessee) for providing plasmids pDL001, pDL004 and pDL006, and Dr. Mike Young (Aberystwyth University, UK) for providing E. coli CA434. We thank Ms. Sheng Dong for constructing C. pasteurianum SD-1, and thank Drs. Michael Pyne and C. Perry Chou (University of Waterloo, Canada) for providing research materials and technical assistance for working on C. pasteurianum. We thank Dr. Daniel Schell (National Renewable Energy Laboratory) for providing the corn stover hydrolysates. We also thank Drs. Raymond P. Henry, Michael E. Miller, Nannan Liu, Evert C. Duin, and Brendan Higgins (all from Auburn University) for allowing us to access their equipment.

\section{Author contributions}

J.F., Y.W. and I.B. designed the experiments. J.F., J. Zhang, Y.M., Shangjun Wang, N.G., H.W., P.W., Y.G., Z.T.Z. and M.C. performed the experiments. Y.F. and H.H. performed the techno-economic analysis (TEA). J.F., Y.F., H.H. and Y.W. drafted the paper. P.J.B., Y.G., J. Zhou, D.J. and X.W.L. contributed to improve the figures. I.B., M.C., Shuning Wang, X.W.L. and Z.S. contributed to the paper revision. All authors read and approved the paper.

\section{Competing interests}

Auburn University has filed a patent application (Application number: PCT/US20/ 66452) covering the engineered Clostridium strains and related bioprocesses for fatty acid ester production as described in this article. Y.W., J.F. and Y.M. are listed as inventors. The other authors claim no competing interests. 


\section{Additional information}

Supplementary information The online version contains supplementary material available at https://doi.org/10.1038/s41467-021-24038-3.

Correspondence and requests for materials should be addressed to H.H. or Y.W.

Peer review information Nature Communications thanks George Bennett, Kurt Rosentrater, Philippe Soucaille and other, anonymous, reviewers for their contributions to the peer review of this work.

Reprints and permission information is available at http://www.nature.com/reprints

Publisher's note Springer Nature remains neutral with regard to jurisdictional claims in published maps and institutional affiliations. (c) (1) Open Access This article is licensed under a Creative Commons Attribution 4.0 International License, which permits use, sharing, adaptation, distribution and reproduction in any medium or format, as long as you give appropriate credit to the original author(s) and the source, provide a link to the Creative Commons license, and indicate if changes were made. The images or other third party material in this article are included in the article's Creative Commons license, unless indicated otherwise in a credit line to the material. If material is not included in the article's Creative Commons license and your intended use is not permitted by statutory regulation or exceeds the permitted use, you will need to obtain permission directly from the copyright holder. To view a copy of this license, visit http://creativecommons.org/ licenses/by/4.0/.

(C) The Author(s) 2021 